\title{
Traditional knowledge and its transmission of wild edibles used by the Naxi in Baidi Village, northwest Yunnan province
}

\author{
Yanfei Geng ${ }^{1,2}$, Yu Zhang ${ }^{1}$, Sailesh Ranjitkar ${ }^{1,3}$, Huyin Huai ${ }^{4}$ and Yuhua Wang ${ }^{1 *}$
}

\begin{abstract}
Background: The collection and consumption of wild edibles is an important part in livelihood strategies throughout the world. There is an urgent need to document and safeguard the wild food knowledge, especially in remote areas. The aims of this study are to accomplish detailed investigation of wild edibles used by the Naxi in Baidi village and evaluate them to identify innovative organic food products. Also, we aim to explore the characteristics of distribution and transmission of the traditional knowledge (TK) on wild edibles among the Naxi.
\end{abstract}

Methods: Data was collected through a semi-structured interview of key informants above the age of 20 years, chosen carefully by a snowball sampling. The interviews were supplemented by free lists and participatory observation methods. Informants below 20 years were interviewed to test their knowledge of traditional practices. A quantitative index like Cultural Importance Index (Cl) was used to evaluate the relative importance of the different wild edibles. Linear regression and $t$-test were performed to test variation in the TK among the informants of different age groups and genders.

Results: Altogether 173 wild edible plant species belonging to 76 families and 139 genera were recorded in the study. Cardamine macrophylla, C. tangutorum and Eutrema yunnanense, have traditionally been consumed as an important supplement to the diet, particularly during food shortages as wild vegetables. The age was found to have a significant effect on TK, but there was no significant difference between male and female informant in knowledge abundance. The traditional food knowledge was dynamic and affected by social factors. Also, it was descending partly among younger generations in Baidi.

Conclusion: Baidi village is a prime example of a rapidly changing community where local traditions compete with modern ways of life. Overall, this study provides a deeper understanding of the Naxi peoples' knowledge on wild edibles. Some wild edibles might have an interesting dietary constituent, which need in-depth studies. Such detail studies can help to promote the market in one hand and protect TK in the other. Protecting TK from disappearing in succeeding generations is necessary, and understanding the dynamics of TK is one important solution to this dilemma.

Keywords: Knowledge dynamics, Quantitative index, Organic food products, Naxi people, Gender

\section{Background}

Wild plants have gained renewed interest in recent years, and the tradition of gathering wild plants continues to the present day $[1,2]$. The collection and consumption of wild edibles is an important part of livelihood strategies throughout the world [3]. Wild food also is an essential supplement to the local people's daily

\footnotetext{
* Correspondence: wangyuhua@mail.kib.ac.cn

'Key Laboratory of Economic Plants and Biotechnology, Kunming Institute of

Botany, Chinese Academy of Sciences, Kunming 650201, China

Full list of author information is available at the end of the article
}

nutrition in developing countries $[2,4,5]$. Schunko and Vogl [6] mentioned that collection and use of wild edibles are not only part of the cultural history of a region but also are part of people's local identity, pride, and traditions. Moreover, wild foods can contribute to overcoming periods of food scarcity, and dishes made of wild foods can be functional foods [6]. Wild plant sources and their use are under severe threat as a result of economic globalization, environmental degradation and cultural homogenization [7]. There is an urgent need to document the traditional knowledge of plant uses and 
conserve its habitat [7-9], especially where it is not yet completely lost [10]. Wild edibles are not an exception to this fact. It is important to document local knowledge before it vanishes along with the knowledgeable people, in the sense that it is slowly disappearing with the demise of those who have traditionally upheld it [11].

China is a fascinating and significant arena for studies on wild food use traditions, particularly Yunnan province [12]. Northwest Yunnan is one of biodiversity hotspots and is home to many minority groups. Some ethnobotanical researchers have documented wild edibles used by different minorities of this region [13-18].

The Naxi people, one of the main ethnic groups in northwest Yunnan, have accumulated rich knowledge on using wild edibles. Baidi Village (Sanba Naxi Nationality Township, Shangri-La City, Deqing Prefecture) is located in $27^{\circ} 30^{\prime} \mathrm{N}$ to $27^{\circ} 28^{\prime} \mathrm{N}$ and $100^{\circ} 01^{\prime} \mathrm{E}$ to $100^{\circ} 05^{\prime} \mathrm{E}$, the Northwest of Yunnan Province, roughly between the two cities Lijiang and Diqing (Fig. 1). It is 103 kilometers from Shangri-La City and 170 kilometers from Lijiang city. The mountain in its territory belongs to Haba Snow Mountain, Yunling Mountain range. Baidi has an area of $8.26 \mathrm{~km}^{2}$ and reaches an elevation of approximately $4500 \mathrm{~m}$ while networks of streams and rivers including Geji and Yangtze dissect numerous valleys, which make it encompass a rich diversity of plants. The village has 15 sections or groups of the settlement, eight of which belong to the Naxi (Fig. 1). In the northwest of the village, there is a big limestone terrace, Baishuitai (literal meaning white water terrace). Local people believe this place as a shrine and perform various religious activities [19]. It also is a famous scenic spot that attracts the considerable number of tourists all over the world.
Baidi comprises approximately 3000 inhabitants, and the majority of them are the Naxi ethnic minority along with about $25 \%$ of the Han people and the Yi people. The Naxi in Baidi is culturally related to the Lijiang Naxi, but they are usually considered the purest of their race $[20,21]$. Joseph Rock, who is a well-known researcher, studied the Naxi people closely and mentioned that the Naxi in Baidi is the most aboriginal among Naxi, and they follow their old religious customs, which are a mixture of shamanism and the pre-Buddhistic Bon religion of Tibet. There are neither Lama temples nor Chinese temples as in the Lijiang city. The Naxi believes that mountains, rivers, trees, herbs, animals and humans, all have their unique spirits. Among these spirits of nature, the Shu spirits are the most important. According to a Naxi myth, farmland and livestock are in the realm of men while Shu rules the mountains and the rivers. Men frequently invaded the territory of Shu creating hostility and fights between men and Shu. Dongba priests, the mediators with spiritual powers, were then called to regain the harmony between them. They agreed that human beings must worship the Shu god of nature every year, in return Shu would provide men's need from nature and stop assaulting them. In this way, men and Shu lived in harmony afterward [21]. The religion and ceremonies of the Naxi represent the long history of keeping equilibrium between man and nature to guarantee the sustainability of natural resources.

Wild edibles in this article refer to those plants that grow without cultivation, including fungi and lichen, and consumed by Naxi people or local animals. It mostly includes native species growing in their natural habitat, but sometimes managed, as well as introduced species

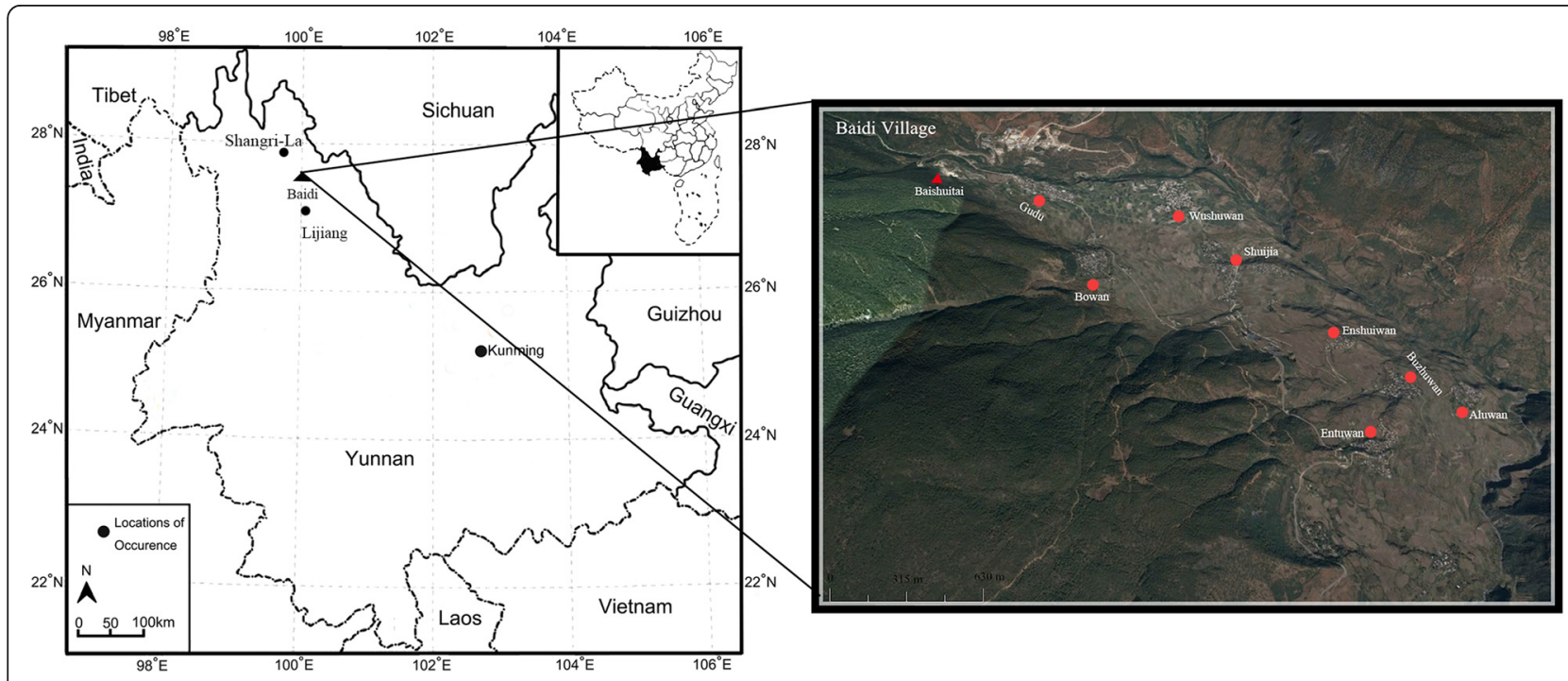

Fig. 1 The location of Baidi village and its small groups 
that have been naturalized [22]. In this paper, we documented angiosperm, gymnosperm, fern, fungi, lichen and algae, which are sources of vegetables, vitamin and functional food, forage, starch and sugar, edible pigments, oil and fats, beverage and honey source.

This study aims to accomplish detailed investigation into wild edibles used by the Naxi in Baidi village and evaluate them to identify innovative organic food products. Also, we aim to explore the distribution of traditional knowledge (TK) and its transmission pathways to the young generation of Naxi.

\section{Methods}

\section{Data collection}

The fieldwork was conducted in 2013 and 2014. Field studies included free lists, semi-structured interviews, and participatory observation. The total of 86 key informants was selected using snowball sampling [23, 24]. The ages of informants ranged from 21 to 91 (mean age 57 years old), and the sex ratio of informants was almost 1:1 (male to female was 42 to 44 ). To that 20 other participants below an age of 20 years (mean age 14 years old) were randomly invited. These youngsters were asked to fill the questionnaire with the purpose of documenting the traditional knowledge transmission.

In the first phase of the field research, participants were invited to list all wild edibles still used on a regular basis, and those were used only in the past. The interviews include the questions that were relevant to document detail information on all wild edibles including the source of knowledge about plant use. Every use report on edible plants included (1) number of useful plants mentioned and their botanical families, (2) most frequently used plant parts, (3) most cited species, (4) ways of consumption and preparation, (5) season of collection, (6) habitats where collected. In the second phase, we collected the wild edibles mentioned above with local gatherers. The participatory observation was utilized to secure the cultural implication of plant gathering, preparation, and distribution of wild edibles. Nomenclature of all vascular plants follows Flora of China [25], and the voucher specimens deposited at the herbarium of the Kunming Institute of Botany, CAS (KUN).

\section{Data analysis}

Ethnobotanical information collected from 86 key informants was properly documented and analyzed. We classified the wild edibles into the following categories based on usage or main chemical composition: carbohydrates, protein, oil and fats, vegetable, vitamin and functional food, beverage, condiments, forage, honey source and chewing and stimulate plants.
To quantify the use frequency of certain species, we calculated the utilization frequency [26], using following formula:

$$
f=\frac{\mathrm{N}_{m}}{\mathrm{~N}_{i}}
$$

In this formula, $f$ represents the utilization frequency, $\mathrm{N}_{\mathrm{m}}$ is the number of informants mentioned certain species, $\mathrm{N}_{\mathrm{i}}$ represents the total number of informants. Higher the value of $f$, the more frequent is the plant used.

Each species mentioned by an informant within one food category was a use report (UR). To determine diversity of uses and the consensus of informants, we used the Cultural Importance Index (CI), which can be mathematically expressed as [27]:

$$
\mathrm{CI}_{\mathrm{s}}=\sum_{\mathrm{u}=\mathrm{u}_{1}}^{\mathrm{u}_{\mathrm{NC}}} \sum_{\mathrm{i}=\mathrm{i}_{1}}^{\mathrm{i}_{\mathrm{N}}} \mathrm{UR}_{\mathrm{ui} / \mathrm{N}}
$$

$\mathrm{N}$ is the total number of informants, and $\mathrm{NC}$ is the total number of use categories. Therefore, the $\mathrm{CI}$ is the sum of the proportion of informants that mention each of the use categories for a given species. This index indicates the spread of the use (number of informants) of each species, as well as the diversity of its uses. Every additional use category is a measure of the relative importance of each plant use [27]. Therefore, multiple uses of a species is an indicator of higher CI value.

Also, the Cultural Food Significance Index (CFSI) was calculated to evaluate the cultural significance of wild edibles using following formula given by Andrea Pieroni [28]:

$$
\begin{aligned}
\mathrm{CFSI}= & \mathrm{QI} \times \mathrm{AI} \times \mathrm{FUI} \times \mathrm{PUI} \times \mathrm{MFFI} \times \mathrm{TSAI} \\
& \times \mathrm{FMRI} \times 10^{-2}
\end{aligned}
$$

This index takes into consideration a wide variety of factors in the evaluation of a specific wild edible. The CFSI include quotation frequency (QI, frequency of quotation index), availability(AI, availability index), typology of the used parts(PUI, parts used index), frequency of use (FUI, frequency of utilization index), kind and number of the food uses (MFFI, multifunctional food use index), taste appreciation (TSAI, taste score appreciation index) and perceived role as food medicine (FMRI, food-medicinal role index). The use of this index allows for exploring the potential wild greens.

To analyze how TK varied according to the characteristics of the different informants, we performed linear regression and $t$-test using $\mathrm{R}$ software (version 3.2.2), taking "Number of edible plants cited by each informant" as the variable to the model. We also consider two entities representing personal data, "ages" (a quantitative variable) and "gender" (a qualitative variable taking a value of male or female). Furthermore, documentation of our 
field investigation was compared with the nutrition information reported in the various relevant literatures.

\section{Results and discussion}

The traditional diet culture of the Baidi village has developed from nomadic lifestyle into an agricultural and pastoral context. Cultivated species play a crucial role in the local diet, but they have a long history of wild edibles gathering. The 86 informants (Fig. 2) of Baidi village reported 173 wild edible species belonging to 76 families and 139 genera (Table 1 ) that they still collecting or had gathered in the past. Table 1 lists the wild edibles mentioned at least by two informants. Botanical and ethnobotanical information about these plants include scientific name, family, voucher or digital photograph number, vernacular name, food categories, part(s) used and mode of consumption (prevalence of use) and collecting habitat (season) [29]. Food categories include carbohydrates, oil and fats, vegetable, vitamin and functional food, beverage, condiments, forage, and honey source. On average, 20.6 edible taxa were listed per informant. The highest number of wild edibles included vegetables (mean - 13.2 species), whereas vitamins and functional foods were frequently used (mean - 7.4). Other categories were less frequent in use such as carbohydrates (mean -0.4), Edible pigments (mean -0.36), Oil and fats (mean - 1.8), Beverage (mean -0.34), Honey source plant (mean -0.23). CI and CFSI values of the wild edibles, except the forage category, cited at least three times were calculated (Table 1).

\section{Diversity of wild edibles}

Almost all major groups of wild plants in Baidi village have edible members that are reported to have been used by the indigenous Naxi people. Exceptions to the bryophytes, documented wild edibles include algae, lichen, fungi, fern, gymnosperm and angiosperm (Table 2). Most of the documented species were angiosperm with 126 species belonging to 53 families. Rosaceae was the biggest family with 18 wild edibles (Fig. 3), whereas 32 families contained only one edible plant species. Fungi was the second largest group containing 37 species representing 17 families. Gymnosperm included one species (one family), fern four species (two families), lichen three species (two families), and algae two species (one family). About one-sixth of 173 wild edibles were included in more than one food category, as listed in Table 3. As to the collecting habitats [29], most of these plants were collected from the wild populations nearby the village. It was also common that there was a small-scale cultivation of wild plants in the home gardens and all the space surrounding human habitations. Different plant parts were used as a source of food in Baidi village, but the most used parts were different depending on the purpose of the foods (such as forage and food medicines). Leaves, fruits, and the complete aerial parts were the mostly consumed by humans while the animals consumed leaves.

\section{Wild vegetables}

This group was the biggest food category with 75 edible species belonging to 40 families. Russulaceae belonged

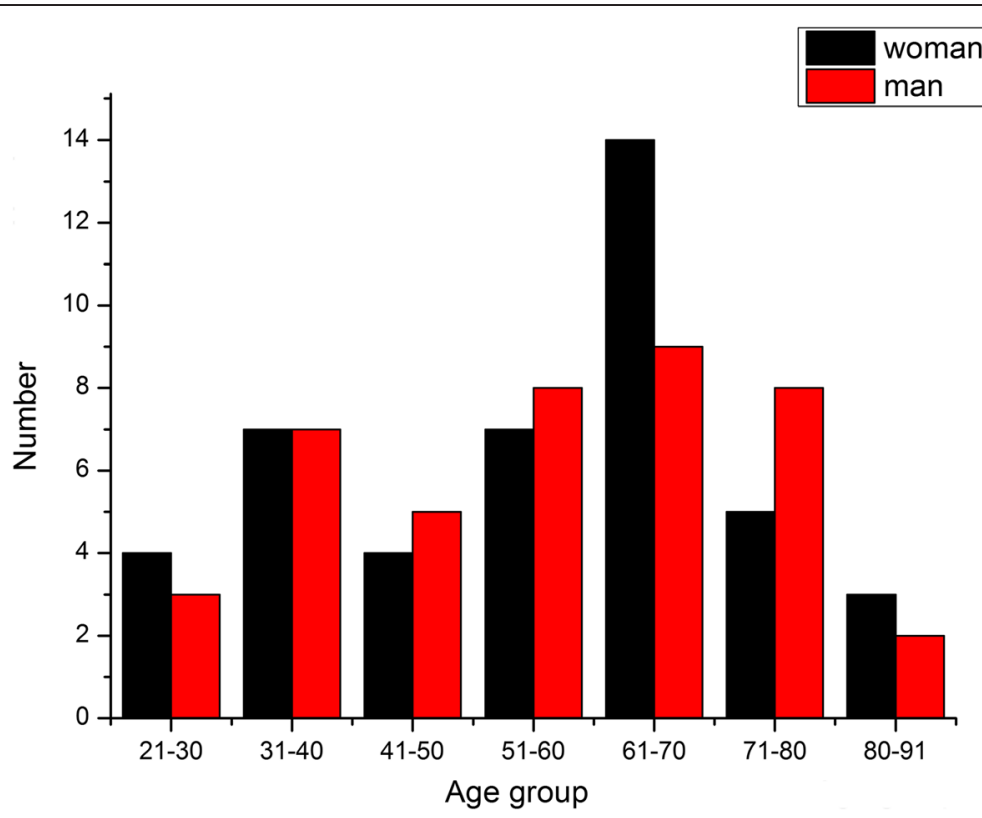

Fig. 2 The age structure of 86 key informants 
Table 1 Inventory of wild edibles gathered and consumed in the Baidi village

\begin{tabular}{|c|c|c|c|c|c|c|c|c|c|c|}
\hline Taxon & Family & $\begin{array}{l}\text { Vernacular } \\
\text { name }\end{array}$ & Food categories & $\begin{array}{l}\text { Part(s) used and mode of consumption } \\
\text { (prevalence of use } \mathrm{e}^{\mathrm{a}} \text { ) }\end{array}$ & $\begin{array}{l}\text { Collecting habitat } \\
\text { (season) }\end{array}$ & $\begin{array}{l}\text { Voucher } \\
\text { number }\end{array}$ & FC & $f$ & $\mathrm{Cl}$ & CFSI \\
\hline \multicolumn{11}{|l|}{ Angiosperma } \\
\hline $\begin{array}{l}\text { Acorus gramineus Sol. } \\
\text { ex Aiton }\end{array}$ & Acoraceae & & $\begin{array}{l}\text { vitamine \& functional } \\
\text { food }\end{array}$ & Rhizomes, boiled in water without garnish (TC). & AE(all seasons) & P1408 & & & & \\
\hline Amaranthus sp. & Amaranthaceae & & vegetable & Leaves, fried (TC). & SC-CA-UA (spring) & 0354 & & & & \\
\hline Chenopodium album $\mathrm{L}$. & Amaranthaceae & mulv & vegetable & Leaves, fried (TC). & $\begin{array}{l}\text { CA-UA (spring and } \\
\text { summer) }\end{array}$ & 0151 & & & & \\
\hline Kochia scoparia (L.) Schrad. & Amaranthaceae & & vegetable & Leaves, fried (TC). & $\begin{array}{l}\text { CA-UA (spring and } \\
\text { summer) }\end{array}$ & P1413 & & & & \\
\hline Allium sp. & Amaryllidaceae & gu & $\begin{array}{l}\text { edible condiments, } \\
\text { vegetable }\end{array}$ & Leaves, fried (TC). & $\begin{array}{l}\text { FO (spring and } \\
\text { summer) }\end{array}$ & 0355 & 40 & 0.47 & 0.47 & 24.00 \\
\hline $\begin{array}{l}\text { Pistacia weinmanniifolia J. } \\
\text { Poiss. ex Franch. }\end{array}$ & Anacardiaceae & yizhu & $\begin{array}{l}\text { vitamine \& functional } \\
\text { food }\end{array}$ & Fruits, eaten raw (AB). & $\begin{array}{l}\text { FO-CA-UA(summer } \\
\text { and autumn) }\end{array}$ & 0055 & 16 & 0.19 & 0.19 & 5.40 \\
\hline $\begin{array}{l}\text { Ligusticum sinense cv. } \\
\text { Chuanxiong S. H. Qiu \& et al. }\end{array}$ & Apiaceae & & $\begin{array}{l}\text { vitamine \& functional } \\
\text { food }\end{array}$ & Roots, boiled in water (TC). & FO(all seasons) & & & & & \\
\hline Oenanthe javanica (BI.) DC. & Apiaceae & zen axi & vegetable & Leaves, fried (TC). & AE(all seasons) & 0045 & 31 & 0.36 & 0.36 & 27.90 \\
\hline $\begin{array}{l}\text { Cynanchum auriculatum Royle } \\
\text { ex Wight }\end{array}$ & Apocynaceae & niezi & vegetable & Leaves and stems, boiled in water (AB). & SC-UA (all seasons) & 0088 & & & & \\
\hline Marsdenia sp. & Apocynaceae & Lubei & vegetable & Leaves and stems, boiled in water (AB). & $\begin{array}{l}\text { SC (spring and } \\
\text { summer) }\end{array}$ & 0234 & & & & \\
\hline $\begin{array}{l}\text { Amorphophallus konjac K. } \\
\text { Koch }\end{array}$ & Araceae & Bulei & carbohydrates & $\begin{array}{l}\text { Tubers, dried, smashed and boiled in water for } \\
\text { making curd (TC). }\end{array}$ & FO-CA-SC (autumn) & 0052 & & & & \\
\hline Arisaema elephas Buchet & Araceae & Babaxiluo & $\begin{array}{l}\text { forage, vitamine \& } \\
\text { functional food }\end{array}$ & $\begin{array}{l}\text { Roots, boiled in water (TC). Leaves, eaten raw as } \\
\text { forage (TC). }\end{array}$ & $\begin{array}{l}\text { SC-CA-UA (all } \\
\text { seasons) }\end{array}$ & 0048 & & & & \\
\hline $\begin{array}{l}\text { Arisaema erubescens (Wall.) } \\
\text { Schott }\end{array}$ & Araceae & Rihaxiluo & $\begin{array}{l}\text { forage, vitamine \& } \\
\text { functional food }\end{array}$ & $\begin{array}{l}\text { Roots, boiled in water (TC). Leaves, eaten raw as } \\
\text { forage (TC). }\end{array}$ & $\begin{array}{l}\text { SC-CA-UA (all } \\
\text { seasons) }\end{array}$ & 0095 & & & & \\
\hline $\begin{array}{l}\text { Asparagus cochinchinensis } \\
\text { (Lour.) Merr. }\end{array}$ & Asparagaceae & Laosha & $\begin{array}{l}\text { vitamine \& functional } \\
\text { food }\end{array}$ & Roots, boiled in water (TC). & $\begin{array}{l}\text { FO-SC-UA (all } \\
\text { seasons) }\end{array}$ & 0047 & & & & \\
\hline $\begin{array}{l}\text { Maianthemum japonicum (A. } \\
\text { Gray) La Frankie }\end{array}$ & Asparagaceae & Abu & vegetable & Leaves, fried (TC). & $\begin{array}{l}\text { FO (spring and } \\
\text { summer) }\end{array}$ & 0011 & 53 & 0.62 & 0.62 & 55.65 \\
\hline Arctium lappa L. & Asteraceae & Elaba & $\begin{array}{l}\text { vegetable, vitamine } \\
\& \text { functional food }\end{array}$ & Roots, stewed (TC). & $\begin{array}{l}\text { SC-CA-U A (all } \\
\text { seasons) }\end{array}$ & 0258 & 4 & 0.05 & 0.05 & 7.02 \\
\hline $\begin{array}{l}\text { Artemisia sieversiana Ehrhart } \\
\text { ex Willd. }\end{array}$ & Asteraceae & & $\begin{array}{l}\text { forage, vitamine \& } \\
\text { functional food }\end{array}$ & $\begin{array}{l}\text { Whole plant, boiled in water (AB). Aerial part, } \\
\text { eaten raw as forage (TC). }\end{array}$ & $\begin{array}{l}\text { FO-SC-CA-UA } \\
\text { (spring, summer } \\
\text { and autumn) }\end{array}$ & 0137 & & & & \\
\hline Carpesium cernuum L. & Asteraceae & La men ga & $\begin{array}{l}\text { forage, vitamine \& } \\
\text { functional food }\end{array}$ & $\begin{array}{l}\text { Whole plant, boiled in water (TC). Aerial part, } \\
\text { eaten raw as forage (TC). }\end{array}$ & SC-UA (all seasons) & 0299 & & & & \\
\hline
\end{tabular}


Table 1 Inventory of wild edibles gathered and consumed in the Baidi village (Continued)

\begin{tabular}{|c|c|c|c|c|c|c|c|c|c|c|}
\hline Carpesium sp. & Asteraceae & La men ga & $\begin{array}{l}\text { forage, vitamine \& } \\
\text { functional food }\end{array}$ & $\begin{array}{l}\text { Whole plant, boiled in water (TC). Aerial part, } \\
\text { eaten raw as forage (TC). }\end{array}$ & SC-UA (all seasons) & 0150 & & & & \\
\hline Cichorium intybus L. & Asteraceae & & vegetable, forage & Leaves, fried (TC). & $\begin{array}{l}\text { SC-CA-UA (all } \\
\text { seasons) }\end{array}$ & P1407 & & & & \\
\hline $\begin{array}{l}\text { Cirsium lidjiangense Petr. \& } \\
\text { Hand.-Mazz. }\end{array}$ & Asteraceae & Raqiku & $\begin{array}{l}\text { vegetable, vitamine } \\
\& \text { functional food }\end{array}$ & Roots, stewed (TC). & $\begin{array}{l}\text { SC-CA-UA (all } \\
\text { seasons) }\end{array}$ & 0260 & 3 & 0.03 & 0.03 & 5.27 \\
\hline Galinsoga parviflora Cav. & Asteraceae & $\begin{array}{l}\text { Munukepei; } \\
\text { Youcong }\end{array}$ & forage & Aerial part, eaten raw (TC). & $\begin{array}{l}\text { CA (spring, summer, } \\
\text { autumn) }\end{array}$ & 0020 & & & & \\
\hline $\begin{array}{l}\text { Hippolytia delavayi (Franch. ex } \\
\text { W. W. Smith) C. Shih }\end{array}$ & Asteraceae & Bunasi & $\begin{array}{l}\text { vitamine \& functional } \\
\text { food }\end{array}$ & Roots, boiled in water (TC). & FO (all seasons) & 0114 & & & & \\
\hline Leibnitzia anandria (L.) Turcz. & Asteraceae & Mumeicidei & forage & Aerial part, eaten raw or boiled in water (AB). & $\begin{array}{l}\text { CA (spring, summer, } \\
\text { autumn) }\end{array}$ & 0061 & & & & \\
\hline Sigesbeckia orientalis L. & Asteraceae & Umeiheiba & forage & Aerial part, eaten raw or boiled in water (TC). & $\begin{array}{l}\text { CA (spring, summer, } \\
\text { autumn) }\end{array}$ & 0101 & & & & \\
\hline Sonchus oleraceus L. & Asteraceae & Umeisennier & vegetable & Leaves, fried (TC). & CA-UA (spring) & P1420 & & & & \\
\hline $\begin{array}{l}\text { Taraxacum mongolicum } \\
\text { Hand.-Mazz. }\end{array}$ & Asteraceae & Pugongying & $\begin{array}{l}\text { vegetable, vitamine } \\
\& \text { functional food }\end{array}$ & Whole plant, boiled in water (TC). & $\begin{array}{l}\text { SC-CA-UA (all } \\
\text { seasons) }\end{array}$ & 0189 & 70 & 0.81 & 0.85 & 157.50 \\
\hline Begonia grandis Dryand. & Begoniaceae & Akangzi & vegetable & Tender leaves and stems, eaten raw (AB). & $\begin{array}{l}\text { FO-CA-UA(summer } \\
\text { and autumn) }\end{array}$ & 0087 & & & & \\
\hline Berberis sp. & Berberidaceae & Ciilv & $\begin{array}{l}\text { vitamine \& functional } \\
\text { food }\end{array}$ & Fruits, eaten raw (TC). & $\begin{array}{l}\text { FO-CA-UA(summer } \\
\text { and autumn) }\end{array}$ & 0007 & 4 & 0.05 & 0.05 & 1.35 \\
\hline $\begin{array}{l}\text { Cynoglossum amabile Stapf \& } \\
\text { J. R. Drumm. }\end{array}$ & Boraginaceae & & forage & Aerial part, eaten raw or boiled in water (AB). & $\begin{array}{l}\text { CA(spring, summer, } \\
\text { autumn) }\end{array}$ & 0064 & & & & \\
\hline Ehretia dicksonii Hance & Boraginaceae & Buna & $\begin{array}{l}\text { forage, vitamine \& } \\
\text { functional food }\end{array}$ & Fruits, eaten raw (AB). Leaves, as forage (AB). & SC-UA (summer) & 0207 & 4 & 0.05 & 0.05 & 1.35 \\
\hline $\begin{array}{l}\text { Capsella bursa-pastoris (L.) } \\
\text { Medik. }\end{array}$ & Brassicaceae & & vegetable & Leaves, fried (TC). & SC-CA-UA (spring) & 0198 & & & & \\
\hline Cardamine macrophylla Willd. & Brassicaceae & You & vegetable & Leaves, fried (TC). & $\begin{array}{l}\text { FO (spring and } \\
\text { summer) }\end{array}$ & 0266 & 76 & 0.88 & 0.94 & 205.20 \\
\hline $\begin{array}{l}\text { Cardamine tangutorum O. E. } \\
\text { Schulz }\end{array}$ & Brassicaceae & You & vegetable & Leaves, fried (TC). & $\begin{array}{l}\text { FO(spring and } \\
\text { summer) }\end{array}$ & 0353 & 76 & 0.88 & 0.94 & 205.20 \\
\hline Eutrema yunnanense Franch. & Brassicaceae & Bei & vegetable, forage & Leaves, fried (TC). Eaten raw by animals. & $\begin{array}{l}\text { FO (spring and } \\
\text { summer) }\end{array}$ & 0352 & 73 & 0.85 & 0.85 & 65.70 \\
\hline Nasturtium officinale R. Br. & Brassicaceae & $\begin{array}{l}\text { Shuicai, } \\
\text { Xiyangcai }\end{array}$ & vegetable & Leaves, fried (CC). & AE(all seasons) & 0166 & 45 & 0.52 & 0.52 & 206.72 \\
\hline Thlaspi arvense L. & Brassicaceae & Jucu & $\begin{array}{l}\text { oil \& fats, vitamine \& } \\
\text { functional food }\end{array}$ & $\begin{array}{l}\text { Seeds, dried and boiled in water (AB). Whole } \\
\text { plant, boiled in water as functional food (AB). }\end{array}$ & SC-UA (summer) & 0129 & & & & \\
\hline Adenophora stricta Miq. & Campanulaceae & Apudada & $\begin{array}{l}\text { vitamine \& functional } \\
\text { food, vegetable }\end{array}$ & $\begin{array}{l}\text { Roots, stewed in meat (TC). Leaves, eaten raw } \\
\text { (TC). }\end{array}$ & CA(all seasons) & 0038 & 38 & 0.44 & 0.45 & 106.88 \\
\hline
\end{tabular}


Table 1 Inventory of wild edibles gathered and consumed in the Baidi village (Continued)

\begin{tabular}{|c|c|c|c|c|c|c|c|c|c|c|}
\hline Cannabis sativa $\mathrm{L}$. & Cannabaceae & Samei & oil \& fats & Seeds, dried and boiled in water (AB). & $\begin{array}{l}\text { SC-CA-UA (summer } \\
\text { and autumn) }\end{array}$ & P1422 & 67 & 0.78 & 0.78 & 24.12 \\
\hline Dipsacus asper Wall. ex DC. & Caprifoliaceae & & $\begin{array}{l}\text { vitamine \& functional } \\
\text { food }\end{array}$ & Roots, boiled in water (TC). & $\begin{array}{l}\text { FO-SC-CA-UA(all } \\
\text { seasons) }\end{array}$ & P1421 & & & & \\
\hline Sambucus adnata Wall. ex DC. & Caprifoliaceae & Shousi & $\begin{array}{l}\text { vitamine \& functional } \\
\text { food }\end{array}$ & Whole plant, boiled in water (TC). & FO-SC(all seasons) & & 3 & 0.03 & 0.03 & 1.01 \\
\hline Sambucus javanica Blume & Caprifoliaceae & Munongzi & $\begin{array}{l}\text { vitamine \& functional } \\
\text { food }\end{array}$ & Whole plant, boiled in water (TC). & $\begin{array}{l}\text { SC-CA-UA (all } \\
\text { seasons) }\end{array}$ & 0227 & & & & \\
\hline Valeriana jatamansi Jones & Caprifoliaceae & Matixiang & $\begin{array}{l}\text { vegetable, vitamine } \\
\& \text { functional food }\end{array}$ & Whole plant, stewed (TC). & $\begin{array}{l}\text { SC-CA-UA (all } \\
\text { seasons) }\end{array}$ & 0041 & 67 & 0.78 & 0.90 & 120.60 \\
\hline Viburnum betulifolium Batalin & Adoxaceae & Efuni & $\begin{array}{l}\text { vitamine \& functional } \\
\text { food }\end{array}$ & Fruits, eaten raw (TC). & $\begin{array}{l}\text { FO-CA-UA(summer } \\
\text { and autumn) }\end{array}$ & 0122 & 30 & 0.35 & 0.35 & 12.15 \\
\hline $\begin{array}{l}\text { Viburnum cylindricum Buch.- } \\
\text { Ham. ex D. Don }\end{array}$ & Adoxaceae & & oil \& fats & Seeds, dried and boiled in water (AB). & FO-SC (winter) & 0035 & & & & \\
\hline $\begin{array}{l}\text { Viburnum foetidum var. } \\
\text { ceanothoides (C. H. Wright) } \\
\text { Handel-Mazzetti }\end{array}$ & Adoxaceae & Ciifuni & $\begin{array}{l}\text { vitamine \& functional } \\
\text { food }\end{array}$ & Fruits, eaten raw (TC). & $\begin{array}{l}\text { FO-CA-UA(summer } \\
\text { and autumn) }\end{array}$ & 0213 & 30 & 0.35 & 0.35 & 12.15 \\
\hline Cuscuta chinensis Lam. & Convolvulaceae & Mulupabie & $\begin{array}{l}\text { vegetable, vitamine } \\
\& \text { functional food }\end{array}$ & Whole plant, boiled in water (TC). & $\begin{array}{l}\text { SC-CA-UA (all } \\
\text { seasons) }\end{array}$ & 0156 & & & & \\
\hline Cornus capitata Wall. & Cornaceae & Laka & $\begin{array}{l}\text { vitamine \& functional } \\
\text { food }\end{array}$ & Fruits, eaten raw (TC). & $\begin{array}{l}\text { FO-CA-UA(summer } \\
\text { and autumn) }\end{array}$ & 0086 & 52 & 0.60 & 0.60 & 14.04 \\
\hline Cyperus sp. & Cyperaceae & Wongdanzi & forage & Aerial part, eaten raw or boiled in water (TC). & $\begin{array}{l}\text { CA(spring, summer } \\
\text { and autumn) }\end{array}$ & & & & & \\
\hline $\begin{array}{l}\text { Dioscorea deltoidea Wall. ex } \\
\text { Griseb. }\end{array}$ & Dioscoreaceae & $\begin{array}{l}\text { Rua ba; } \\
\text { Luanba }\end{array}$ & carbohydrates & Tubers, dried and boiled in water (TC). & FO-CA-SC (autumn) & 0094 & 7 & 0.08 & 0.08 & 4.73 \\
\hline $\begin{array}{l}\text { Dioscorea yunnanensis Prain \& } \\
\text { Burkill }\end{array}$ & Dipsacaceae & & $\begin{array}{l}\text { vitamine \& functional } \\
\text { food }\end{array}$ & Roots, boiled in water (TC). & $\begin{array}{l}\text { FO-SC-CA-UA (all } \\
\text { seasons) }\end{array}$ & P1409 & & & & \\
\hline Diospyros lotus L. & Ebenaceae & Tazhu & $\begin{array}{l}\text { vitamine \& functional } \\
\text { food }\end{array}$ & Fruits, eaten raw (TC). & $\begin{array}{l}\text { FO-CA-UA(summer } \\
\text { and autumn) }\end{array}$ & P1417 & 3 & 0.03 & 0.03 & 1.01 \\
\hline Elaeagnus umbellata Thunb. & Elaeagnaceae & & vegetable & Fruits, eaten raw (TC). & SC-UA (autumn) & 0211 & & & & \\
\hline Hippophae rhamnoides L. & Elaeagnaceae & Zhu & beverage & Fruits, fermented for sour taste (AB). & FO-SC (autumn) & & & & & \\
\hline Pyrola atropurpurea Franch. & Ericaceae & & forage & Aerial part, eaten raw or boiled in water (TC). & $\begin{array}{l}\text { FO (spring, summer } \\
\text { and autumn) }\end{array}$ & & & & & \\
\hline Vaccinium fragile Franch. & Ericaceae & Anmiximi & $\begin{array}{l}\text { vitamine \& functional } \\
\text { food }\end{array}$ & Fruits, eaten raw (AB). & $\begin{array}{l}\text { FO-CA-UA(summer } \\
\text { and autumn) }\end{array}$ & 0021 & & & & \\
\hline Bauhinia sp. & Fabaceae & Huangrekei & forage & Leaves, eaten raw or boiled in water (TC). & $\begin{array}{l}\text { FO (spring, summer } \\
\text { and autumn) }\end{array}$ & 0142 & & & & \\
\hline Cassia sp. & Fabaceae & Wujibaba & forage & $\begin{array}{l}\text { Leaves, eaten raw or boiled in water for livestocks } \\
\text { (TC). }\end{array}$ & $\begin{array}{l}\text { FO (spring, summer } \\
\text { and autumn) }\end{array}$ & 0319 & & & & \\
\hline
\end{tabular}


Table 1 Inventory of wild edibles gathered and consumed in the Baidi village (Continued)

\begin{tabular}{|c|c|c|c|c|c|c|c|c|c|c|}
\hline Lespedeza sp. & Fabaceae & Fushibeibei & forage & Leaves, eaten raw or boiled in water (AB). & $\begin{array}{l}\text { FO (spring, summer } \\
\text { and autumn) }\end{array}$ & 0100 & & & & \\
\hline $\begin{array}{l}\text { Lespedeza thunbergii subsp. } \\
\text { elliptica (Benth. ex Maxim.) H. } \\
\text { Ohashi }\end{array}$ & Fabaceae & & forage & Leaves, eaten raw or boiled in water (AB). & $\begin{array}{l}\text { FO(spring, summer, } \\
\text { autumn) }\end{array}$ & 0091 & & & & \\
\hline Medicago Iupulina L. & Fabaceae & Mosu & forage & Aerial part, eaten raw or boiled in water (TC). & $\begin{array}{l}\text { CA(spring, summer } \\
\text { and autumn) }\end{array}$ & 0239 & & & & \\
\hline $\begin{array}{l}\text { Piptanthus nepalensis (Hook.) } \\
\text { Sweet }\end{array}$ & Fabaceae & Murekei & forage & Leaves, eaten raw or boiled in water (TC). & $\begin{array}{l}\text { FO(spring, summer } \\
\text { and autumn) }\end{array}$ & 0105 & & & & \\
\hline Trifolium repens $\mathrm{L}$. & Fabaceae & & forage & Aerial part, eaten raw (TC). & CA(all seasons) & P1415 & & & & \\
\hline Quercus sp. & Fagaceae & Laba & forage & Tender Leaves, eaten raw or boiled in water (TC). & FO (all seasons) & 0098 & & & & \\
\hline Gentiana rigescens Franch. & Gentianaceae & Yinini; Zii & $\begin{array}{l}\text { vitamine \& functional } \\
\text { food }\end{array}$ & Whole plant, boiled in water (TC). & FO-SC (all seasons) & 0326 & & & & \\
\hline Helwingia chinensis Batalin & Helwingiaceae & Ninahagubii & vegetable & Tender leaves, fried (AB). & $\begin{array}{l}\text { FO (spring and } \\
\text { summer) }\end{array}$ & 0215 & 4 & 0.05 & 0.05 & 0.90 \\
\hline $\begin{array}{l}\text { Hypericum forrestii (Chitt.) N. } \\
\text { Robson }\end{array}$ & Hypericaceae & Muwaniba & honey source plant & Flowers, sucked (AB). & SC-CA-UA (summer) & 0243 & & & & \\
\hline Itea yunnanensis Franch. & Iteaceae & Piejulu & forage & Tender leaves, eaten raw (TC). & $\begin{array}{l}\text { FO-CA-UA(summer } \\
\text { and autumn) }\end{array}$ & 0077 & & & & \\
\hline Juglans cathayensis Dode & Juglandaceae & Gudu & oil \& fats & Seeds, dried and boiled in water (AB). & $\begin{array}{l}\text { FO-SC-CA-UA } \\
\text { (autumn and } \\
\text { winter) }\end{array}$ & P1412 & 67 & 0.78 & 0.80 & 20.35 \\
\hline Dracocephalum sp. & Lamiaceae & Bingba & forage & Aerial part, eaten raw or boiled in water (AB). & $\begin{array}{l}\text { FO (spring, summer } \\
\text { and autumn) }\end{array}$ & 0039 & & & & \\
\hline $\begin{array}{l}\text { Elsholtzia strobilifera (Benth.) } \\
\text { Benth. }\end{array}$ & Lamiaceae & & edible condiments & Seeds, dried, for seasoning (AB). & $\begin{array}{l}\text { SC-CA-UA (autumn } \\
\text { and winter) }\end{array}$ & 0192 & & & & \\
\hline Mentha canadensis $L$. & Lamiaceae & Angzhi & $\begin{array}{l}\text { vegetable, edible } \\
\text { condiments }\end{array}$ & $\begin{array}{l}\text { Tender leaves and stems, fried, or cold and } \\
\text { dressed with sauce (TC). }\end{array}$ & CA-UA (all seasons) & 0012 & 43 & 0.50 & 0.50 & 169.31 \\
\hline Origanum vulgare L. & Lamiaceae & Kedu & edible condiments & Seeds and leaves, dried, for seasoning (AB). & $\begin{array}{l}\text { SC-CA-UA (autumn } \\
\text { and winter) }\end{array}$ & 0058 & & & & \\
\hline Salvia trijuga Diels & Lamiaceae & & $\begin{array}{l}\text { vitamine \& functional } \\
\text { food }\end{array}$ & Roots, boiled in water (TC). & $\begin{array}{l}\text { FO-SC-UA (all } \\
\text { seasons) }\end{array}$ & 0119 & & & & \\
\hline Streptolirion volubile Edgew. & Commelinaceae & Mailexu & forage & Aerial part, eaten raw (TC). & $\begin{array}{l}\text { CA(spring, summer } \\
\text { and autumn) }\end{array}$ & 0030 & & & & \\
\hline Malva verticillata $\mathrm{L}$. & Malvaceae & & carbohydrates & Tubers, dried and boiled in water (TC). & FO-CA-SC (autumn) & 0152 & & & & \\
\hline $\begin{array}{l}\text { Ficus sarmentosa Buch.-Ham. } \\
\text { ex Sm. }\end{array}$ & Moraceae & Kesulu & $\begin{array}{l}\text { vitamine \& functional } \\
\text { food }\end{array}$ & Fruits, eaten raw (TC). & $\begin{array}{l}\text { FO-CA-UA(summer } \\
\text { and autumn) }\end{array}$ & 0040 & 3 & 0.03 & 0.03 & 1.01 \\
\hline $\begin{array}{l}\text { Morus mongolica (Bureau) C. } \\
\text { K. Schneid. }\end{array}$ & Moraceae & Ciilu & $\begin{array}{l}\text { vitamine \& functional } \\
\text { food }\end{array}$ & Fruits, eaten raw (TC). & $\begin{array}{l}\text { FO-CA-UA(summer } \\
\text { and autumn) }\end{array}$ & 0132 & & & & \\
\hline
\end{tabular}


Table 1 Inventory of wild edibles gathered and consumed in the Baidi village (Continued)

\begin{tabular}{|c|c|c|c|c|c|c|c|c|c|c|}
\hline Epipactis mairei Schltr. & Orchidaceae & $\mathrm{aba}$ & forage & Aerial part, eaten raw or boiled in water (AB). & $\begin{array}{l}\text { FO(spring, summer } \\
\text { and autumn) }\end{array}$ & 0026 & & & & \\
\hline Habenaria sp. & Orchidaceae & & $\begin{array}{l}\text { vitamine \& functional } \\
\text { food }\end{array}$ & Tubers, boiled in water (AB). & FO (autumn) & 0037 & & & & \\
\hline Oxalis acetosella $\mathrm{L}$. & Oxalidaceae & Tuolaibaba & forage & Aerial part, eaten raw or boiled in water (TC). & $\begin{array}{l}\text { CA (spring, summer } \\
\text { and autumn) }\end{array}$ & 0313 & & & & \\
\hline Plantago asiatica $\mathrm{L}$. & Plantaginaceae & Umeiheizhou & $\begin{array}{l}\text { vegetable, vitamine } \\
\& \text { functional food }\end{array}$ & Whole plant, boiled in water (TC). & $\begin{array}{l}\text { SC-CA-UA (all } \\
\text { seasons) }\end{array}$ & 0049 & 64 & 0.74 & 0.78 & 144.00 \\
\hline Avena fatua $\mathrm{L}$. & Poaceae & Wongdaba & carbohydrates forage & $\begin{array}{l}\text { Seeds, dried, smashed and fried (TC). Whole plant } \\
\text { for animal (TC). }\end{array}$ & $\begin{array}{l}\text { CA (summer and } \\
\text { autumn) }\end{array}$ & P1419 & & & & \\
\hline $\begin{array}{l}\text { Catabrosa aquatica (L.) P. } \\
\text { Beauv. }\end{array}$ & Poaceae & Zii & forage & Aerial part, eaten raw or boiled in water (TC). & $\begin{array}{l}\text { CA (spring, summer } \\
\text { and autumn) }\end{array}$ & 0256 & & & & \\
\hline $\begin{array}{l}\text { Echinochloa crusgalli (L.) P. } \\
\text { Beauv. }\end{array}$ & Poaceae & Bai & carbohydrates & Seeds, dried (AB). & $\begin{array}{l}\text { SC-CA-UA (summer, } \\
\text { autumn and winter) }\end{array}$ & 0146 & & & & \\
\hline Phyllostachys glauca McClure & Poaceae & Zhusun & vegetable & Young shoots, fried (TC). & $\begin{array}{l}\text { SC-CA-UA (spring } \\
\text { and early summer) }\end{array}$ & 0154 & 5 & 0.06 & 0.06 & 1.13 \\
\hline Setaria viridis (L.) P. Beauv. & Poaceae & Kucuzii & forage & Aerial part, eaten raw or boiled in water (TC). & $\begin{array}{l}\text { CA (spring, summer } \\
\text { and autumn) }\end{array}$ & & & & & \\
\hline $\begin{array}{l}\text { Fagopyrum dibotrys (D. Don) } \\
\text { H.Hara }\end{array}$ & Polygonaceae & Saidiku & forage & Aerial part, eaten raw or boiled in water (TC). & CA (all seasons) & 0015 & & & & \\
\hline $\begin{array}{l}\text { Fagopyrum gracilipes (Hemsl.) } \\
\text { Dammer ex Diels }\end{array}$ & Polygonaceae & Niarlagulepo & forage & Aerial part, eaten raw or boiled in water (TC). & $\begin{array}{l}\text { CA (spring, summer } \\
\text { and autumn) }\end{array}$ & 0141 & & & & \\
\hline Oxyria sinensis Hemsl. & Polygonaceae & Huaji & vegetable, forage & $\begin{array}{l}\text { Young shoots, eaten raw by people (AB). Leaves, } \\
\text { eaten by animals (TC). }\end{array}$ & $\begin{array}{l}\text { SC-CA-UA (spring, } \\
\text { summer and } \\
\text { autumn) }\end{array}$ & 0176 & 10 & 0.12 & 0.23 & 15.19 \\
\hline $\begin{array}{l}\text { Polygonum capitatum Buch.- } \\
\text { Ham. ex D. Don }\end{array}$ & Polygonaceae & Niaorla & forage & Aerial part, eaten raw (TC). & $\begin{array}{l}\text { CA (spring, summer } \\
\text { and autumn) }\end{array}$ & 0144 & & & & \\
\hline Polygonum paleaceum Wall. & Polygonaceae & Yeku & $\begin{array}{l}\text { vitamine \& functional } \\
\text { food }\end{array}$ & Roots, boiled in water (TC). & FO (all seasons) & & & & & \\
\hline $\begin{array}{l}\text { Polygonum runcinatum Buch.- } \\
\text { Ham. ex D. Don }\end{array}$ & Polygonaceae & Lagasidi & vegetable & Leaves, fried (TC). & $\begin{array}{l}\text { SC-CA-UA (spring, } \\
\text { summer and } \\
\text { autumn) }\end{array}$ & 0237 & 6 & 0.07 & 0.07 & 4.68 \\
\hline Rumex acetosa $\mathrm{L}$. & Polygonaceae & Lagasidi & vegetable & Young shoots, eaten raw (AB). & $\begin{array}{l}\text { SC-CA-UA (spring, } \\
\text { summer and } \\
\text { autumn) }\end{array}$ & 0236 & & & & \\
\hline Myrsine africana $\mathrm{L}$. & Primulaceae & Lagancii & $\begin{array}{l}\text { vitamine \& functional } \\
\text { food }\end{array}$ & Fruits, eaten raw (AB). & $\begin{array}{l}\text { FO-CA-UA(summer } \\
\text { and autumn) }\end{array}$ & 0076 & & & & \\
\hline Clematis armandii Franch. & Ranunculaceae & Ehake & vegetable & Young shoots, fried (TC). & SC-CA-UA (spring) & 0163 & & & & \\
\hline Clematis ranunculoides Franch. & Ranunculaceae & Umeijuzi & forage & Aerial part, eaten raw or boiled in water (TC). & $\begin{array}{l}\text { CA (spring, summer } \\
\text { and autumn) }\end{array}$ & 0046 & & & & \\
\hline
\end{tabular}


Table 1 Inventory of wild edibles gathered and consumed in the Baidi village (Continued)

\begin{tabular}{|c|c|c|c|c|c|c|c|c|c|c|}
\hline $\begin{array}{l}\text { Thalictrum aquilegiifolium L. } \\
\text { var. sibiricum Regel \& Tiling }\end{array}$ & Ranunculaceae & Renuba & forage & Aerial part, eaten raw or boiled in water (TC). & $\begin{array}{l}\text { CA(spring, summer } \\
\text { and autumn) }\end{array}$ & 0042 & & & & \\
\hline $\begin{array}{l}\text { Ziziphus montana W. W. } \\
\text { Smith }\end{array}$ & Rhamnaceae & Cipa & $\begin{array}{l}\text { vitamine \& functional } \\
\text { food }\end{array}$ & Fruits, eaten raw (TC). & $\begin{array}{l}\text { FO-CA-UA(summer } \\
\text { and autumn) }\end{array}$ & 0349 & 17 & 0.20 & 0.20 & 5.74 \\
\hline $\begin{array}{l}\text { Amygdalus davidiana } \\
\text { (Carrière) de Vos ex Henry }\end{array}$ & Rosaceae & Buji,buka & $\begin{array}{l}\text { vitamine \& functional } \\
\text { food }\end{array}$ & Fruits, eaten raw (TC). & $\begin{array}{l}\text { FO-CA-UA(summer } \\
\text { and autumn) }\end{array}$ & 0217 & & & & \\
\hline $\begin{array}{l}\text { Cerasus cerasoides (Buch.- } \\
\text { Ham. ex D. Don) S. Y. Sokolov }\end{array}$ & Rosaceae & & $\begin{array}{l}\text { vitamine \& functional } \\
\text { food }\end{array}$ & Fruits, eaten raw (TC). & $\begin{array}{l}\text { FO-CA-UA(summer } \\
\text { and autumn) }\end{array}$ & P1402 & & & & \\
\hline $\begin{array}{l}\text { Docynia delavayi (Franch.) C. } \\
\text { K. Schneid. }\end{array}$ & Rosaceae & Sibu & $\begin{array}{l}\text { vitamine \& functional } \\
\text { food }\end{array}$ & Fruits, eaten raw (TC). & $\begin{array}{l}\text { FO-CA-UA(summer } \\
\text { and autumn) }\end{array}$ & & 51 & 0.59 & 0.59 & 11.48 \\
\hline $\begin{array}{l}\text { Fragaria nilgerrensis Schltdl. ex } \\
\text { J. Gay }\end{array}$ & Rosaceae & $\begin{array}{l}\text { Anmenbuzi; } \\
\text { Alibuji; } \\
\text { Ameibuji }\end{array}$ & $\begin{array}{l}\text { vitamine \& functional } \\
\text { food }\end{array}$ & Fruits, eaten raw (TC). & $\begin{array}{l}\text { FO-CA-UA(summer } \\
\text { and autumn) }\end{array}$ & P1414 & & & & \\
\hline Fragaria vesca $\mathrm{L}$. & Rosaceae & Ameibuji & $\begin{array}{l}\text { vitamine \& functional } \\
\text { food }\end{array}$ & Fruits, eaten raw (TC). & $\begin{array}{l}\text { FO-CA-UA(summer } \\
\text { and autumn) }\end{array}$ & 0226 & 14 & 0.16 & 0.16 & 3.15 \\
\hline Malus rockii Rehder & Rosaceae & & $\begin{array}{l}\text { vitamine \& functional } \\
\text { food }\end{array}$ & Fruits, eaten raw (TC). & $\begin{array}{l}\text { FO-CA-UA(summer } \\
\text { and autumn) }\end{array}$ & & & & & \\
\hline $\begin{array}{l}\text { Malus yunnanensis (Franch.) } \\
\text { C.K. Schneid. }\end{array}$ & Rosaceae & Lvba & $\begin{array}{l}\text { vitamine \& functional } \\
\text { food }\end{array}$ & Fruits, eaten raw (TC). & $\begin{array}{l}\text { FO-CA-UA(summer } \\
\text { and autumn) }\end{array}$ & 0210 & & & & \\
\hline $\begin{array}{l}\text { Osteomeles schwerinae C. K. } \\
\text { Schneid. }\end{array}$ & Rosaceae & Dazhu & $\begin{array}{l}\text { vitamine \& functional } \\
\text { food }\end{array}$ & Fruits, eaten raw $(A B)$. & $\begin{array}{l}\text { FO-CA-UA(summer } \\
\text { and autumn) }\end{array}$ & 0346 & 18 & 0.21 & 0.21 & 4.05 \\
\hline $\begin{array}{l}\text { Potentilla Kleiniana Wight \& } \\
\text { Arn. }\end{array}$ & Rosaceae & & forage & Aerial part, eaten raw or boiled in water (TC). & $\begin{array}{l}\text { CA (spring, summer } \\
\text { and autumn) }\end{array}$ & 0079 & & & & \\
\hline Prinsepia utilis Royle & Rosaceae & Chuda & $\begin{array}{l}\text { vitamine \& functional } \\
\text { food, beverage, oil \& } \\
\text { fats }\end{array}$ & $\begin{array}{l}\text { Fruits, eaten raw (AB). Seeds, smashed and boiled } \\
\text { in water for oil (CC). Leaves, for making functional } \\
\text { tea (TC). }\end{array}$ & $\begin{array}{l}\text { SC-CA-UA(spring, } \\
\text { summer and } \\
\text { autumn) }\end{array}$ & 0159 & 45 & 0.52 & 0.57 & 177.69 \\
\hline $\begin{array}{l}\text { Pyracantha angustifolia } \\
\text { (Franch.) C. K. Schneid. }\end{array}$ & Rosaceae & $\begin{array}{l}\text { Anmilaximi; } \\
\text { Saigulu; } \\
\text { Youlubuzhu }\end{array}$ & $\begin{array}{l}\text { vitamine \& functional } \\
\text { food }\end{array}$ & Fruits, eaten raw (AB). & $\begin{array}{l}\text { FO-CA-UA(summer } \\
\text { and autumn) }\end{array}$ & 0229 & 9 & 0.10 & 0.10 & 2.63 \\
\hline $\begin{array}{l}\text { Pyracantha fortuneana } \\
\text { (Maxim.) H. L. Li }\end{array}$ & Rosaceae & Abalugu & $\begin{array}{l}\text { vitamine \& functional } \\
\text { food }\end{array}$ & Fruits, eaten raw $(A B)$. & $\begin{array}{l}\text { FO-CA-UA(summer } \\
\text { and autumn) }\end{array}$ & 0004 & 9 & 0.10 & 0.10 & 2.63 \\
\hline $\begin{array}{l}\text { Pyrus pashia Buch.-Ham. ex D. } \\
\text { Don }\end{array}$ & Rosaceae & & $\begin{array}{l}\text { vitamine \& functional } \\
\text { food }\end{array}$ & Fruits, eaten raw (TC). & $\begin{array}{l}\text { FO-CA-UA(summer } \\
\text { and autumn) }\end{array}$ & P1410 & & & & \\
\hline Rosa sp. & Rosaceae & Haducii & $\begin{array}{l}\text { vitamine \& functional } \\
\text { food }\end{array}$ & Fruits, eaten raw (TC). & $\begin{array}{l}\text { FO-CA-UA(summer } \\
\text { and autumn) }\end{array}$ & 0128 & & & & \\
\hline $\begin{array}{l}\text { Rubus biflorus Buch.-Ham. ex } \\
\text { Sm. }\end{array}$ & Rosaceae & Cipaaha & $\begin{array}{l}\text { vitamine \& functional } \\
\text { food }\end{array}$ & Fruits, eaten raw (TC). & $\begin{array}{l}\text { FO-CA-UA(summer } \\
\text { and autumn) }\end{array}$ & 0265 & 29 & 0.34 & 0.34 & 9.79 \\
\hline \multirow[t]{2}{*}{ Rubus sp. } & Rosaceae & Ciinaaha & $\begin{array}{l}\text { vitamine \& functional } \\
\text { food }\end{array}$ & Fruits, eaten raw (TC). & $\begin{array}{l}\text { FO-CA-UA(summer } \\
\text { and autumn) }\end{array}$ & 0172 & 29 & 0.34 & 0.34 & 9.79 \\
\hline & Rosaceae & Emaiji & & Fruits, eaten raw (TC). & & 0274 & 48 & 0.56 & 0.56 & 135.00 \\
\hline
\end{tabular}


Table 1 Inventory of wild edibles gathered and consumed in the Baidi village (Continued)

\begin{tabular}{|c|c|c|c|c|c|c|c|c|c|c|}
\hline $\begin{array}{l}\text { Sorbus hemsleyi (C. K. } \\
\text { Schneid.) Rehder }\end{array}$ & & & $\begin{array}{l}\text { vitamine \& functional } \\
\text { food }\end{array}$ & & $\begin{array}{l}\text { FO-CA-UA(summer } \\
\text { and autumn) }\end{array}$ & & & & & \\
\hline $\begin{array}{l}\text { Sorbus hupehensis C. K. } \\
\text { Schneid. }\end{array}$ & Rosaceae & Yumaiji & $\begin{array}{l}\text { vitamine \& functional } \\
\text { food }\end{array}$ & $\begin{array}{l}\text { Fruits, eaten raw (TC). Dried and pounded to } \\
\text { powder to cure high blood pressure (TC). }\end{array}$ & $\begin{array}{l}\text { FO-CA-UA(summer } \\
\text { and autumn) }\end{array}$ & 0275 & 48 & 0.56 & 0.56 & 135.00 \\
\hline Rubia membranacea Diels & Rubiaceae & & forage & Aerial part, eaten raw or boiled in water (TC). & $\begin{array}{l}\text { CA(spring, summer } \\
\text { and autumn) }\end{array}$ & 0121 & & & & \\
\hline Zanthoxylum armatum DC. & Rutaceae & & edible condiments & Fruit shells, dried, for seasoning (TC). & FO-SC-CA (autumn) & 0139 & & & & \\
\hline $\begin{array}{l}\text { Zanthoxylum bungeanum } \\
\text { Maxim. }\end{array}$ & Rutaceae & Yehuajiao & edible condiments & Fruit shells, dried, for seasoning (TC). & FO-SC-CA (autumn) & 0078 & 5 & 0.06 & 0.08 & 13.78 \\
\hline Houttuynia cordata Thunb. & Saururaceae & Arunaha; Azina & $\begin{array}{l}\text { vitamine \& functional } \\
\text { food, vegetable }\end{array}$ & $\begin{array}{l}\text { Tender leaves, stems and roots, fried, or cold and } \\
\text { dressed with sauce (TC). Leaves, boiled in water } \\
\text { (TC). }\end{array}$ & CA-UA (all seasons) & 0044 & 74 & 0.86 & 1.21 & 2164.50 \\
\hline Schisandra sp. & Schisandraceae & & beverage & Fruits, for making liqueur (TC) & FO (spring) & & & & & \\
\hline $\begin{array}{l}\text { Debregeasia orientalis C. J. } \\
\text { Chen }\end{array}$ & Urticaceae & Pimi & vegetable & Young shoots and flower, eaten raw (AB). & SC-CA-UA (spring) & 0143 & 12 & 0.14 & 0.14 & 8.10 \\
\hline Verbena officinalis $\mathrm{L}$. & Verbenaceae & & $\begin{array}{l}\text { vegetable, vitamine } \\
\& \text { functional food }\end{array}$ & Whole plant, boiled in water (TC). & $\begin{array}{l}\text { SC-CA-UA (all } \\
\text { seasons) }\end{array}$ & 0014 & 11 & 0.13 & 0.13 & 96.80 \\
\hline Viola sp. & Violaceae & $\begin{array}{l}\text { Lagagudu; } \\
\text { Lagaseimei }\end{array}$ & $\begin{array}{l}\text { vitamine \& functional } \\
\text { food }\end{array}$ & Fruits, eaten raw (AB). & $\begin{array}{l}\text { FO-CA-UA(summer } \\
\text { and autumn) }\end{array}$ & 0180 & 4 & 0.05 & 0.05 & 1.35 \\
\hline $\begin{array}{l}\text { Ampelopsis delavayana } \\
\text { Planch. }\end{array}$ & Vitaceae & Gaiha & $\begin{array}{l}\text { vitamine \& functional } \\
\text { food }\end{array}$ & Fruits, eaten raw (TC). & $\begin{array}{l}\text { FO-CA-UA (summer } \\
\text { and autumn) }\end{array}$ & 0084 & & & & \\
\hline \multicolumn{11}{|l|}{ Gymnosperm } \\
\hline Pinus armandi Franch. & Pinaceae & Situo & carbohydrates & Seeds, eaten raw (TC). & FO-SC (autumn) & 0250 & 10 & 0.12 & 0.12 & 2.25 \\
\hline \multicolumn{11}{|l|}{ Fern } \\
\hline Equisetum hyemale L. & Equisetaceae & & forage & Aerial part, eaten raw or boiled in water (AB). & $\begin{array}{l}\text { CA (spring, summer } \\
\text { and autumn) }\end{array}$ & & & & & \\
\hline Adiantum sp. & Pteridaceae & & forage & Aerial part, eaten raw or boiled in water (TC). & $\begin{array}{l}\text { FO (spring, summer } \\
\text { and autumn) }\end{array}$ & 0303 & & & & \\
\hline $\begin{array}{l}\text { Pteridium aquilinum var. } \\
\text { latiusculum (Desv.) Underw. } \\
\text { ex Heller. }\end{array}$ & Pteridaceae & Ade & vegetable & Leaves, fried (TC). & $\begin{array}{l}\text { FO (spring and } \\
\text { summer) }\end{array}$ & 0222 & 38 & 0.44 & 0.44 & 51.3 \\
\hline $\begin{array}{l}\text { Pteridium revolutum (Blume) } \\
\text { Nakai }\end{array}$ & Pteridaceae & Angzhide & vegetable & Leaves, fried (TC). & $\begin{array}{l}\text { FO (spring and } \\
\text { summer) }\end{array}$ & 0267 & 38 & 0.44 & 0.44 & 51.3 \\
\hline \multicolumn{11}{|l|}{ Mushroom } \\
\hline Auricularia sp. & Auriculariaceae & Muer & vegetable & Fruit body, fried (TC). & $\begin{array}{l}\text { FO (summer and } \\
\text { autumn) }\end{array}$ & P1416 & & & & \\
\hline Boletus edulis Bull. & Boletaceae & Chumugulu & vegetable & Fruit body, stewed, fried (TC). & $\begin{array}{l}\text { FO (summer and } \\
\text { autumn) }\end{array}$ & 0342 & 8 & 0.09 & 0.09 & 4.80 \\
\hline
\end{tabular}


Table 1 Inventory of wild edibles gathered and consumed in the Baidi village (Continued)

\begin{tabular}{|c|c|c|c|c|c|c|c|c|c|c|}
\hline Tylopilus balloui (Peck) Sing & Boletaceae & Niuganjun & vegetable & Fruit body, stewed, fried (TC). & $\begin{array}{l}\text { FO (summer and } \\
\text { autumn) }\end{array}$ & & & & & \\
\hline Tylopilus sp. & Boletaceae & Bamu & vegetable & Fruit body, stewed, fried (TC). & $\begin{array}{l}\text { FO (summer and } \\
\text { autumn) }\end{array}$ & & 10 & 0.12 & 0.12 & 5.20 \\
\hline Cantharellus cibarius Fr. & Cantharellaceae & Jiyoujun & vegetable & Fruit body, stewed, fried (TC). & $\begin{array}{l}\text { FO (summer and } \\
\text { autumn) }\end{array}$ & P1405 & 10 & 0.12 & 0.12 & 6.00 \\
\hline $\begin{array}{l}\text { Cordyceps sobolifera (Hill.) } \\
\text { Berk. et Br. }\end{array}$ & Clavicipitaceae & Chongcao & $\begin{array}{l}\text { vitamine \& functional } \\
\text { food }\end{array}$ & Fruit body, stewed (TC). & FO (summer) & & & & & \\
\hline $\begin{array}{l}\text { Entoloma clypeatum (L.) P. } \\
\text { Kumm. }\end{array}$ & Entolomataceae & Yiwojun & vegetable & Fruit body, fried (TC). & $\begin{array}{l}\text { FO (summer and } \\
\text { autumn) }\end{array}$ & P1406 & & & & \\
\hline Gomphus sp. & Gomphaceae & Labajun & vegetable & Fruit body, stewed, fried (TC). & $\begin{array}{l}\text { FO (summer and } \\
\text { autumn) }\end{array}$ & & & & & \\
\hline Hericium sp. & Hericiaceae & Houtoujun & vegetable & Fruit body, stewed (TC). & $\begin{array}{l}\text { FO (spring, summer } \\
\text { and autumn) }\end{array}$ & & 5 & 0.06 & 0.06 & 2.60 \\
\hline Laccaria laccata (Scop.) Cooke & Hydnangiaceae & Tashimu & vegetable & Fruit body, stewed, fried (TC). & $\begin{array}{l}\text { FO (summer and } \\
\text { autumn) }\end{array}$ & 0282 & & & & \\
\hline Hygrophorus sp. & Hygrophoraceae & Huanglasan & vegetable & Fruit body, fried (TC). & FO (autumn) & & 3 & 0.03 & 0.03 & 1.80 \\
\hline Engleromyces sp. & Hypocreaceae & Zhujun & vegetable & Fruit body, dried and stewed (TC). & FO (summer) & & & & & \\
\hline Lentinula sp. & Marasmiaceae & Zhemu & vegetable & Fruit body, fried (TC). & $\begin{array}{l}\text { FO (summer and } \\
\text { autumn) }\end{array}$ & & 6 & 0.07 & 0.07 & 3.60 \\
\hline Morchella esculenta Pers. & Morchellaceae & Aboduoluoluo & vegetable & Fruit body, stewed (TC). & FO (summer) & & & & & \\
\hline Coriolus versicolor L. & Polyporaceae & Lingzhi & $\begin{array}{l}\text { vitamine \& functional } \\
\text { food }\end{array}$ & Fruit body, stewed (TC). & FO(autumn) & P1401 & & & & \\
\hline Polyporus sp. & Polyporaceae & Musi & vegetable & Fruit body, fried (AB). & $\begin{array}{l}\text { FO-SC (spring and } \\
\text { summer) }\end{array}$ & 0278 & 3 & 0.03 & 0.03 & 1.80 \\
\hline $\begin{array}{l}\text { Ramaria rubri-attenuipes R.H. } \\
\text { Petersen \& M. Zang }\end{array}$ & Ramariaceae & Saobajun & vegetable & Fruit body, stewed, fried (TC). & FO (summer) & & 12 & 0.14 & 0.14 & 6.24 \\
\hline Lactaricus sp. 1 & Russulaceae & Wenzhishi & vegetable & Fruit body, stewed, fried (TC). & $\begin{array}{l}\text { FO (spring and } \\
\text { summer) }\end{array}$ & 0338 & 14 & 0.16 & 0.16 & 8.40 \\
\hline Lactaricus sp. 2 & Russulaceae & Baipajun & vegetable & Fruit body, stewed, fried (TC). & $\begin{array}{l}\text { FO (spring and } \\
\text { summer) }\end{array}$ & 0341 & 17 & 0.20 & 0.20 & 10.20 \\
\hline Lactaricus sp. 3 & Russulaceae & Minuka & vegetable & Fruit body, stewed, fried (TC). & $\begin{array}{l}\text { FO (spring and } \\
\text { summer) }\end{array}$ & 0284 & & & & \\
\hline Lactarius hatsudake Tanaka & Russulaceae & Tongbulu & vegetable & Fruit body, stewed, fried (TC). & FO (summer) & 0285 & & & & \\
\hline Lactarius sp. 4 & Russulaceae & Angzhishi & vegetable & Fruit body, stewed, fried (TC). & $\begin{array}{l}\text { FO (spring and } \\
\text { summer) }\end{array}$ & 0288 & & & & \\
\hline Lactarius sp. 5 & Russulaceae & Jucu & vegetable & Fruit body, stewed, fried (TC). & $\begin{array}{l}\text { FO (spring and } \\
\text { summer) }\end{array}$ & 0281 & & & & \\
\hline
\end{tabular}


Table 1 Inventory of wild edibles gathered and consumed in the Baidi village (Continued)

\begin{tabular}{|c|c|c|c|c|c|c|c|c|c|c|}
\hline Russula sp. 1 & Russulaceae & Kaca & vegetable & Fruit body, fried (TC). & $\begin{array}{l}\text { FO (summer and } \\
\text { autumn) }\end{array}$ & 0286 & 9 & 0.10 & 0.10 & 5.40 \\
\hline Russula sp. 2 & Russulaceae & Zhebu & vegetable & Fruit body, fried (TC). & $\begin{array}{l}\text { FO (summer and } \\
\text { autumn) }\end{array}$ & 0340 & 9 & 0.10 & 0.10 & 5.40 \\
\hline Russula sp. 3 & Russulaceae & Huotanjun & vegetable & Fruit body, fried (AB). & FO (summer) & & & & & \\
\hline Russula sp. 4 & Russulaceae & Azimenihu & vegetable & Fruit body, fried (TC). & $\begin{array}{l}\text { FO (summer and } \\
\text { autumn) }\end{array}$ & 0283 & & & & \\
\hline $\begin{array}{l}\text { Russula virescens (schaeff. ex } \\
\text { Zanted) Fr. }\end{array}$ & Russulaceae & Qingtoujun & vegetable & Fruit body, stewed, fried (TC). & FO (summer) & & 4 & 0.05 & 0.05 & 2.40 \\
\hline Thelephora sp. & Thelephoraceae & Ganbajun & vegetable & Fruit body, fried (TC). & FO (summer) & & & & & \\
\hline Tricholoma matsutake Sing & Thelephoraceae & Songmaojun & vegetable & Fruit body, stewed, fried (TC). & FO (autumn) & & & & & \\
\hline Tricholoma sp. & Thelephoraceae & Songmaojun & vegetable & Fruit body, stewed, fried (TC). & FO (autumn) & & & & & \\
\hline Tricholoma sp. & Thelephoraceae & Yumu & vegetable & Fruit body, stewed, fried (TC). & FO (autumn) & & 19 & 0.22 & 0.22 & 41.04 \\
\hline $\begin{array}{l}\text { Lyophyllum fumosum (Pers. : } \\
\text { Fr.) P. D. Orton. }\end{array}$ & Tricholomataceae & Yiwojun & vegetable & Fruit body, fried (TC). & $\begin{array}{l}\text { FO (summer and } \\
\text { autumn) }\end{array}$ & & & & & \\
\hline Lyophyllum sp. & Tricholomataceae & Menzher & vegetable & , stewed (TC). & $\begin{array}{l}\text { FO (summer and } \\
\text { autumn) }\end{array}$ & 0280 & 31 & 0.36 & 0.36 & 22.32 \\
\hline Marasmius sp. & Tricholomataceae & Huangpijun & vegetable & Fruit body, fried (TC). & FO (summer) & & 3 & 0.03 & 0.03 & 1.80 \\
\hline Termitomyces sp. 1 & Tricholomataceae & Mulu & vegetable & $\begin{array}{l}\text { Fruit body, stewed, dried, dried and fried, salted } \\
\text { (TC) }\end{array}$ & $\begin{array}{l}\text { FO (summer and } \\
\text { autumn) }\end{array}$ & P1403 & 36 & 0.42 & 0.42 & 116.64 \\
\hline Termitomyces sp. 2 & Tricholomataceae & Umu & vegetable & $\begin{array}{l}\text { Fruit body, stewed, dried, dried and fried, salted } \\
\text { (TC) }\end{array}$ & $\begin{array}{l}\text { FO (summer and } \\
\text { autumn) }\end{array}$ & P1403 & & & & \\
\hline \multicolumn{11}{|l|}{ Lichen } \\
\hline Lobaria retigera Trevis. & Lobariaceae & & vegetable & $\begin{array}{l}\text { Whole plant, stewed, or cold and dressed with } \\
\text { sauce (AB). }\end{array}$ & FO (all seasons) & 0219 & 42 & 0.49 & 0.49 & 3.47 \\
\hline Lobaria yunnanensis Yoshim & Lobariaceae & & vegetable & $\begin{array}{l}\text { Whole plant, stewed, or cold and dressed with } \\
\text { sauce (AB). }\end{array}$ & FO (all seasons) & 0253 & 42 & 0.49 & 0.49 & 3.47 \\
\hline $\begin{array}{l}\text { Thamnolia vermicularia (Sw.) } \\
\text { Ach. Ex Schae }\end{array}$ & Thamnoliaceae & & beverage & Whole plant, for making tea (TC). & RP (spring) & & & & & \\
\hline \multicolumn{11}{|l|}{ Algae } \\
\hline $\begin{array}{l}\text { Nostoc commune Vaucher ex } \\
\text { Bornet \& Flahault }\end{array}$ & Nostocaceae & Baqi & vegetable & Aerial part, fried (TC). & AE (summer) & 0279 & & & & \\
\hline Nostoc sphaeroids Kutz & Nostocaceae & Bacai i e & vegetable & Aerial part, fried (TC). & FO (summer) & P1411 & & & & \\
\hline
\end{tabular}

Species in inventory are ordered from higher to lower plants, and they are arranged firstly by family taxa and then by genus taxa. Vernacular name of wild edibles are written using Chinese pinyin The types of collecting habitats are based on the characterization proposed by Calabuig (2008)

$F C$ frequency of citation, CFSI cultural food significance index, $\mathrm{Cl}$ cultural importance index

aPrevalence of use: $A B$ Abandoned, $C C$ currently consumed, $T C$ traditionally consumed

${ }^{b}$ Collecting habitat: FO Forests (oak woods, pine woods, etc.); SC Scrublands (Pistacia, etc.); AE Aquatic environments (streams, ditch, wet places, etc.); RP Rock places (rocky slopes, rocks, etc.); CA Cultivated areas

(orchards, farmland, etc.); UA Urban and periurban areas (villages, roads etc.)

Voucher number with $\mathrm{P}$ means voucher photograph number, and the one without $\mathrm{P}$ means voucher specimen number 
Table 2 Number of species and number of families in different plant categories

\begin{tabular}{lll}
\hline Plant categories & Number of families & Number of species \\
\hline Angiosperm & 53 & 126 \\
Gymnospermae & 1 & 1 \\
Fern & 2 & 4 \\
Fungi & 17 & 37 \\
Lichen & 2 & 3 \\
Algae & 1 & 2 \\
Total & 76 & 173 \\
\hline
\end{tabular}

to fungi had the highest number of species (11 species) eaten as vegetables (Fig. 4). Often wild vegetables were cooked in oil or fat or consumed in stews and soups. The most common procedure was to boil them first and then fried with garlic and chilies. The pork fat was common compared to vegetable oil for frying. The consumption of wild vegetable eaten raw was very rare.

The most frequently reported species were Cardamine macrophylla Willd., C. tangutorum O. E. Schulz., Eutrema yunnanense Franch. and Houttuynia cordata Thunb. All of these consumed after frying, except the last one, consumed as a salad with sauce. The first three species grow in the mountains, local people collected these species most often while grazing their cattle and horses during the spring and summer seasons. Houttuynia cordata grew wild in cropland and was collected by the local people when they finished their farm work. These four wild vegetables had been consumed for a long time, especially in
Table 3 Number of species in different food categories

\begin{tabular}{ll}
\hline Food categories & Number of species \\
\hline Vegetable & 75 \\
Vitamin and functional food & 60 \\
Forage & 40 \\
Carbohydrates & 6 \\
Edible pigments & 6 \\
Oil and fats & 5 \\
Beverage & 4 \\
Honey source plant & 1 \\
\hline
\end{tabular}

the time of food shortages, later became the most popular vegetables in Baidi village. Wild gathered vegetables had different chemical composition and nutritional value from cultivated ones, according to Zeghichi [30]. Another two wild vegetables often used in the past, especially in time of food shortage, were the well-known Lobaria retigera Trevis. and L. yunnanensis Yoshim. (laolongpi is a vernacular name for both). These two plants are still consumed in other regions, like the Naxi in Lijiang city. These two species are proved to have high nutritional values such as antioxidant activity [31], but the Naxi in Baidi village abandoned this food tradition because of the unpleasant taste.

Most of the wild vegetables were defined as "bitter", according to the Naxi, who related this to the concept of "healthy". This kind of vegetable was considered "healthy" without any specification. According to Johns [32], such use had cultural significance related to the ingestion rather than taste.

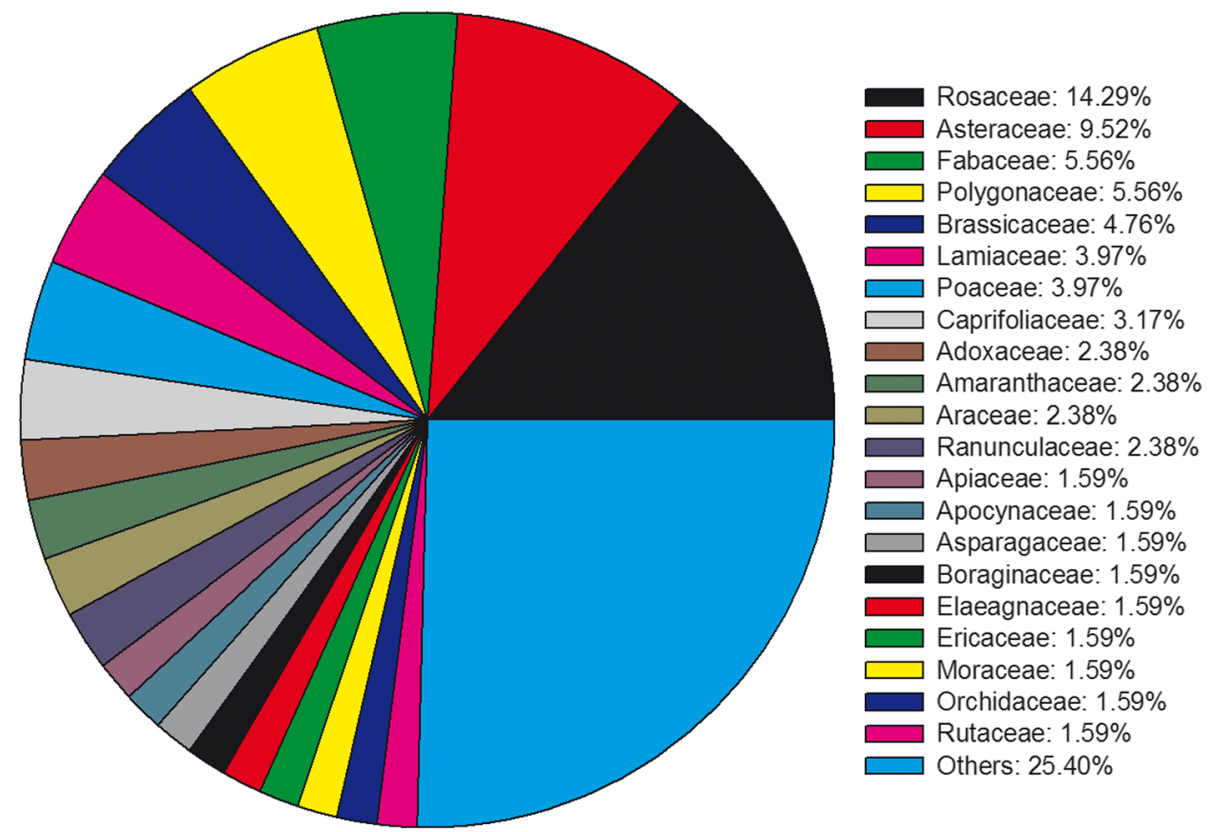

Fig. 3 Family distribution of wild edible plant species of angiosperm category 


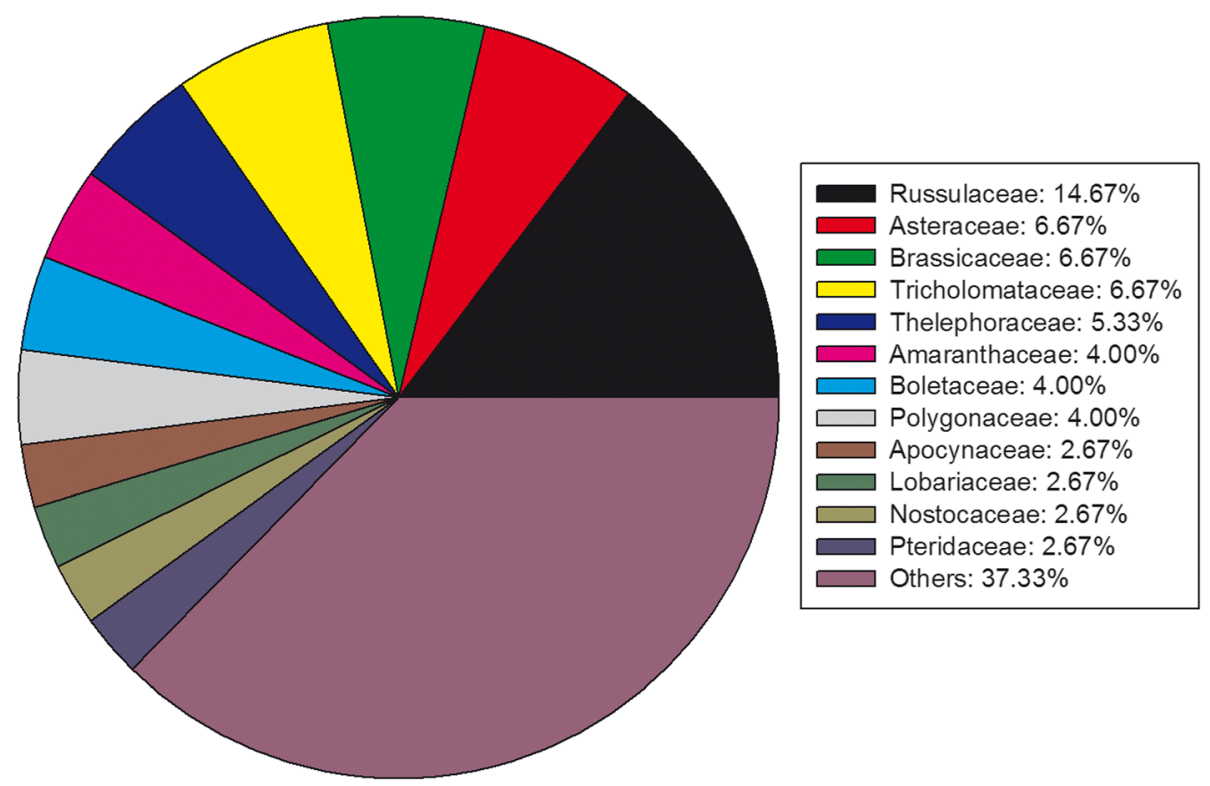

Fig. 4 Percentage of wild vegetables in each family

Mushrooms also played a significant role in the local diet. Of 37 fungi species, most were eaten as vegetables and could be gathered during spring and fall. The mushrooms were consumed while fresh or after drying, and mostly grilled like meat. The harvesting of fungi for markets also had been one of main economic activities in the Baidi village. According to informants, Boletus edulis Bull., Cantharellus cibarius Fr. and Entoloma clypeatum (L.) P. Kumm., for example, were sold to Lijiang city, and to other provinces, such as Guangdong in Southern China. Women and children were the primary collectors of mushrooms. Mushrooms are the source of food in more than 80 countries worldwide, and their commercial harvesting is an important business in many countries, such as Turkey, the USA and Bhutan [33].

\section{Vitamin and functional food}

This food category included mainly wild fruits, which had a high content of vitamins and minerals, and food medicines consumed as both edible plants and medicinal plants. This group, with 60 species, was the second largest regarding the number of wild edibles cited.

Of the 30 wild edible fruits, Rosaceae was the largest family. Most of them did not have market value and sporadically gathered for household consumption, except Malus pumila Mill. and Pyrus pashia Buch.-Ham. ex D. Don. The most frequently eaten fruit was Cornus capitata Wall. for the Naxi. According to Johns [34], wild fruits are more fibrous and contain higher concentrations of vitamins and a greater diversity of secondary compounds compared to the cultivated species. Our study showed that many wild fruits were used as snacks, mainly by children in the past when cultivated fruits were not frequently available. They were probably a good source of vitamins and minerals but have become less important now, such as Rubus biflorus Buch.-Ham. ex Sm.

We documented 33 species belonging to food medicines. It is interesting that food medicines can also be wild vegetables and wild fruits. For example, Houttuynia cordata was delicious salad and antiphlogosis medicine. Similarly, Sorbus hupehensis C. K. Schneid. was tasty fruit and medicine to high blood pressure. Balick and Cox [35] explained aboriginal people do not make a clear distinction between edible and medicinal plants; we documented similar findings in the traditional practice of the Naxi in Baidi village. This kind of practices also exists in other Naxi villages in Shangri-La [15]. Moreover, some food preparations were taken exclusively for medicinal purpose, for example, Habenaria sp. fried with eggs was the most commonly used medicine for a cough [36, 37].

\section{Carbohydrates and edible condiments}

In the past, underground parts of some wild edibles such as Dioscorea oppositifolia that contain a high amount of starch used to be consumed, especially in the time of hardship. We documented six wild edibles used as the source of carbohydrates, out of that two were abandoned, and the remaining four were occasionally consumed. The main reason for the decrease in consumption was the diversity and abundance of cultivated crops in Baidi village. It was very common that wild edibles once frequently consumed in the past were now considered as weeds 
and rarely eaten. Such kind of change in perception has been reported from several places in Turkey, India and Brazil [38-40].

There were only six condiments from the wild source in the diet of Baidi village according to this study. The most often consumed species was Zanthoxylum armatum DC. The use of condiments not only enhances the flavor of certain dishes but also provides preservative and medicinal properties (anti-parasitic) [41].

\section{Oil and fats, beverage and honey source plant}

The Naxi in Baidi used total five wild edibles as a source of oil and fats, of which Juglans mandshurica Maxim. and Cannabis sativa L. were most commonly used. These two species were still widely used to make oil and fats. Similarly, Prinsepia utilis oil, rich in flavonoid and have been proved to have an anti-bacteria effect $[42,43]$, was also frequently used.

A total of four wild edibles recorded were used as the beverage. Fruits of Schisandra sp. were usually soaked in wine, which make the liquor medicinal [44, 45]. Leaves of the three species (Hippophae rhamnoides, Prinsepia utilis and Thamnolia vermicularia), were used to make vinegar and tea. Tea made of Prinsepia utilis has been proved to have significant immunosuppressive and antitumor activity $[46,47]$.
Hypericum forrestii (Chittenden) N. Robson was the only honey source plant. The local name for this species is "muwaniba", which means it blooms during Dragon Boat Festival. This species with bright flowers attracts lots of bees during the flowering season, and local children have the habits of sucking its nectar for a sweet taste.

\section{Forage}

Altogether 40 wild species belong to 20 families were used as animal fodder in Baidi village. According to informants, they divided fodder plants into two groups: cropland group and mountain group based on the habitats. In mountain group, Eutrema yunnanense was the favorite fodder for the cattle. In cropland group, Fagopyrum gracilipes (Hemsl.) Dammer was often intentionally cultivated as animal fodder. Naxi women collected and carried those fodders from the cropland for stall feeding. The fodder plants also included Oxyria sinensis Hemsl. and Cichorium intybus L., the local people once consumed both of these during the food scarcity.

\section{Evaluating and selecting of wild edibles based on traditional wisdom}

Twenty wild edibles were selected (Table 4) using four quantitative indices (FC, f, CI and CFSI). The ranks of

Table 4 Evaluation of wild edibles (except forage category) of the Baidi village using four indices

\begin{tabular}{|c|c|c|c|c|c|c|c|c|c|}
\hline \multirow[t]{2}{*}{ Latin name } & \multirow{2}{*}{$\begin{array}{l}\text { Vernacular } \\
\text { name }\end{array}$} & \multicolumn{4}{|c|}{ Indices } & \multicolumn{4}{|c|}{ Ranking } \\
\hline & & FC & $f$ & $\mathrm{Cl}$ & CFSI & FC & $f$ & $\mathrm{Cl}$ & CFSI \\
\hline Cardamine macrophylla Willd. & You & 76 & 0.88 & 0.94 & 205.20 & 1 & 1 & 2 & 3 \\
\hline Cardamine tangutorum O. E. Schulz & You & 76 & 0.88 & 0.94 & 205.20 & 1 & 1 & 2 & 3 \\
\hline Houttuynia cordata Thunb. & Arunaha; Azina & 74 & 0.86 & 1.21 & 2164.50 & 2 & 2 & 1 & 1 \\
\hline Eutrema yunnanense Franch. & Bei & 73 & 0.85 & 0.85 & 65.70 & 3 & 3 & 4 & 13 \\
\hline Taraxacum mongolicum Hand.-Mazz. & Pugongying & 70 & 0.81 & 0.85 & 157.50 & 4 & 4 & 4 & 6 \\
\hline Cannabis sativa $\mathrm{L}$. & Samei & 67 & 0.78 & 0.78 & 24.12 & 5 & 5 & 6 & 18 \\
\hline Juglans cathayensis Dode & Gudu & 67 & 0.78 & 0.80 & 20.35 & 5 & 5 & 5 & 21 \\
\hline Valeriana jatamansi Jones & Matixiang & 67 & 0.78 & 0.90 & 120.60 & 5 & 5 & 3 & 9 \\
\hline Plantago asiatica $\mathrm{L}$. & Umeiheizhou & 64 & 0.74 & 0.78 & 144.00 & 6 & 6 & 6 & 7 \\
\hline Maianthemum japonicum (A. Gray) La Frankie & Abu & 53 & 0.62 & 0.62 & 55.65 & 7 & 7 & 7 & 14 \\
\hline Cornus capitata Wall. & Laka & 52 & 0.60 & 0.60 & 14.04 & 8 & 8 & 8 & 24 \\
\hline Docynia delavayi (Franch.) C. K. Schneid. & Sibu & 51 & 0.59 & 0.59 & 11.48 & 9 & 9 & 9 & 26 \\
\hline Sorbus hemsleyi (C. K. Schneid.) Rehder & Emaiji & 48 & 0.56 & 0.56 & 135.00 & 10 & 10 & 11 & 8 \\
\hline Sorbus hupehensis C. K. Schneid. & Yumaiji & 48 & 0.56 & 0.56 & 135.00 & 10 & 10 & 11 & 8 \\
\hline Nasturtium officinale R. Br. & Shuicai & 45 & 0.52 & 0.52 & 206.72 & 11 & 11 & 12 & 2 \\
\hline Prinsepia utilis Royle & Chuda & 45 & 0.52 & 0.57 & 177.69 & 11 & 11 & 10 & 4 \\
\hline Mentha canadensis $\mathrm{L}$. & Angzhi & 43 & 0.50 & 0.50 & 169.31 & 12 & 12 & 13 & 5 \\
\hline Lobaria retigera Trevis. & Laolongpi & 42 & 0.49 & 0.49 & 3.47 & 13 & 13 & 14 & 38 \\
\hline Lobaria yunnanensis Yoshim & Laolongpi & 42 & 0.49 & 0.49 & 3.47 & 13 & 13 & 14 & 38 \\
\hline Allium sp. & $\mathrm{Gu}$ & 40 & 0.47 & 0.47 & 24.00 & 14 & 14 & 15 & 19 \\
\hline
\end{tabular}


some species based on different indices were different, indicating that different indices assigned particular importance of the various attributes, such as the multiplicity of uses and taste appreciation [27].

\section{Food botanicals with high $\mathrm{Cl}$ values}

Wild edibles that had high CI values were Houttuynia cordata (1.21), Cardamine macrophylla (0.94), C. tangutorum (0.94), Valeriana jatamansi Jones (0.90) and Eutrema yunnanense (0.85). Whole plants of Houttuynia cordata and Valeriana jatamansi were consumed as functional food having a medicinal property, whereas the others were frequently eaten leafy vegetables.

Wild edibles with high CI values might have an interesting dietary constituent and needed further research. Also, a plant with a low $\mathrm{CI}$ value could be an important plant for a few people [27].

\section{Food botanicals with high CFSI values}

Wild edibles that had high CFSI values had different ranks from those with high $\mathrm{CI}$ values, and they were Houttuynia cordata (2164.50), Nasturtium officinale (206.72), Cardamine macrophylla (205.20), Cardamine tangutorum (205.20) and Prinsepia utilis (177.69). Three of them (Houttuynia cordata, Cardamine tangutorum and Cardamine macrophylla) were also in the front rank when assessed with CI values, but only Houttuynia cordata was positioned the same place when assessed with CFSI and CI values. Eutrema yunnanense growing on the high-elevation mountains ranked $13^{\text {th }}$ with CFSI index, attributed to its low availability index value, multifunctional food use index value and food-medicinal role index value. The local people consumed Eutrema yunnanense only as vegetables, and the collection was often time-consuming due to its mountain-grown habitat. While Nasturtium officinale and Prinsepia utilis were in the front position for their high availability index value, and food-medicinal-role index value respectively.

\section{Traditional wisdom from the Naxi}

Our interview indicated a long history of consumption of Cardamine macrophylla, C. tangutorum and Eutrema yunnanense (Fig. 5). The results of quantitative indices showed that Cardamine macrophylla, C. tangutorum, and Eutrema yunnanense were in front positions. Hence,
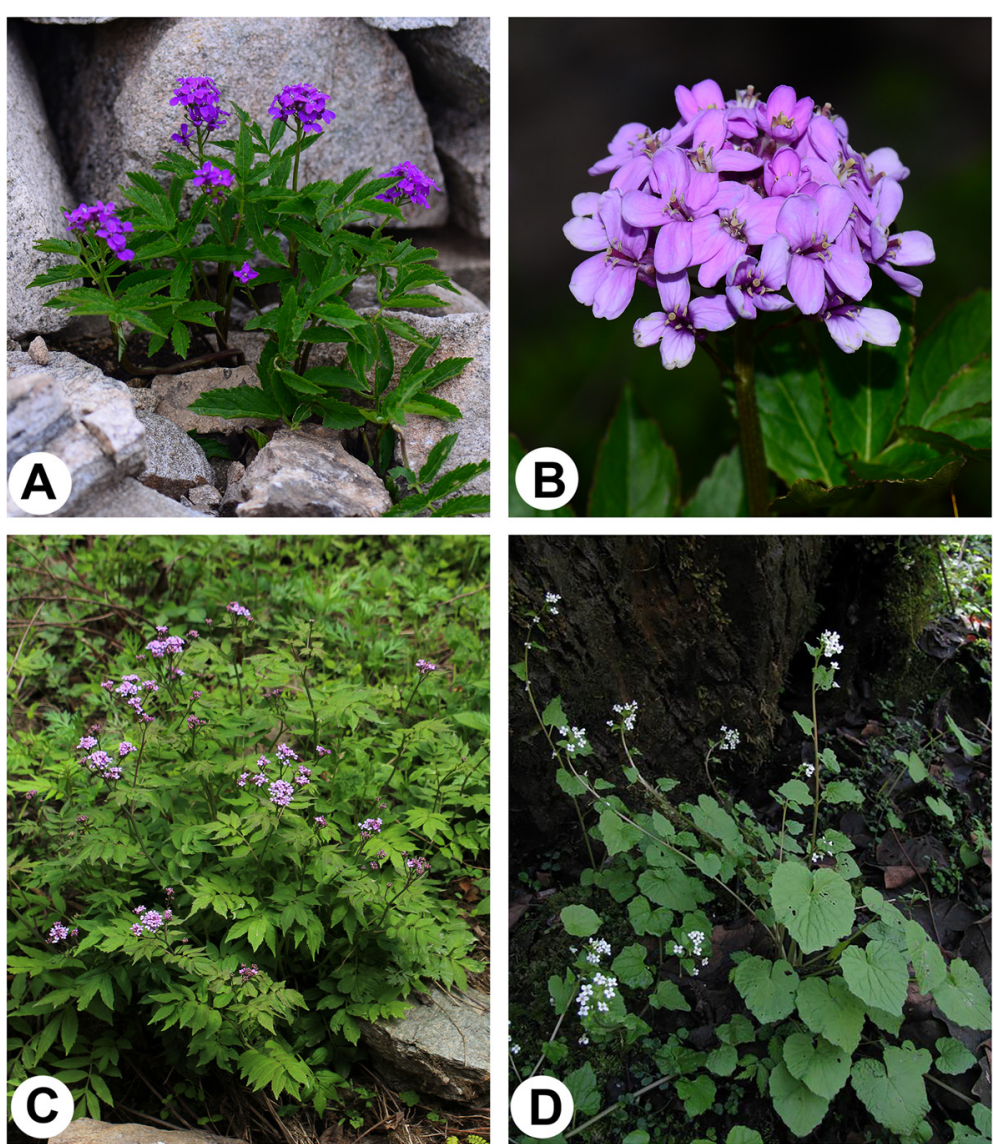

Fig. 5 Three wild edible species with most promising exploitation prospects. a Cardamine tangutorum, whole plant; $\mathbf{b}$. C. tangutorum, inflorescence; c C. macrophylla; d. Eutrema yunnanense. a-c photo by Renbin Zhu, (d) photo by Dahai Zhu 
we selected these three species as most promising organic products.

Relevant literature studies show that high levels of vitamin $\mathrm{C}$, minerals, fibers and protein have been reported in Cardamine macrophylla [48-52]. Also, low concentration of heavy metal has been found in this wild edible species. As its affinity, C. tangutorum, theoretically also had abundant nutrient components. Furthermore, other species of Cardamine are consumed as wild vegetables in Tanzania, India, Poland, United States and Slovakia [53-57]. Eutrema wasabi Maxim. is one of the raw materials of mustard. The species has proved to have anti-bacterial activity and flavor components $[58,59]$, and it has been developed as a condiment for many years by Lijiang Washabi Company Eutrema yunnanense widely consumed in Baidi village seems to be a potential vegetable. Additionally, as an affinity of $E$. wasabi, this species may have a similar chemical component with $E$. wasabi, and consequently use a substitute for $E$. wasabi.

Apart from the consumption in the rural area, the market of these wild edibles was expanded in the nearby city areas in the recent years. However, the scientific research regarding nutritional, phytochemical or phytopharmacological analysis was not conducted on the wild edibles recorded in Baidi village. In the context of increasing interest in the health potential foods, such as functional food and pharmafood, studies on wild edibles regarding the nutritional and medicinal qualities, and as potential alternative crops may be very useful [60]. The resurgence of the interest in the wild edibles was also consistent with a reappraisal of traditional cuisines, for example in European countries and with the general claim for 'natural' foods [61].

\section{Age, gender and knowledge dynamics}

Age, gender, and traditional knowledge

All the informants in Baidi agreed that they consumed less number of wild edibles compared to the previous decades. Our results indicate the younger people almost could not identify, gather and process these species. Similarly, many middle-aged informants regarded the consumption of wild edibles as a symbol of poverty as they consumed these wild edibles during the time of scarcity. However, the gathering of wild edibles, such as Cardamine macrophylla, C. tangutorum and Eutrema yunnanense in the spring still represents a significant role in the daily diet. Overall, the number of wild edibles cited by informants increased with age according to our regression analysis (Fig. 6), even the correlation was weak $(P$ value $<0.01$, the coefficient $=0.19)$. Concurrent to our results, differences in the knowledge of wild food plants and wild edible fungi among different age groups is reported in two valleys of Shaanxi, central China [62]. However, decreasing knowledge trends in youngsters are common as in the case of other parts of the Himalayas [7]. A study in a Caribbean village finds that the older the people, the less they are affected by external influences [63]. In Baidi, many young people have migrated to other cities in Yunnan to search for employment and education in recent decades. According to our informants, such migration severely disrupted the transfer of local wild edibles knowledge between generations and led to the loss of TK.

The $t$-test results showed that there were no significant differences between females and males $(P$ value $=$ 0.361 ), even the number of species cited by women and men in different age groups fluctuated all the time. According to Pfeiffer and Butz [64], gender is a critical variable that influences local knowledge distribution, as it is highly correlated with other sociocultural factors including occupation, education, resource access, and social status and networks. Women tend to know more traditional knowledge $[65,66]$ because of the sociocultural factors mentioned above. Women are usually unemployed in the rural areas, dedicating themselves to the household and subsistence activities, and they combine this information with their cultural background as well as external knowledge to improve their subsistence

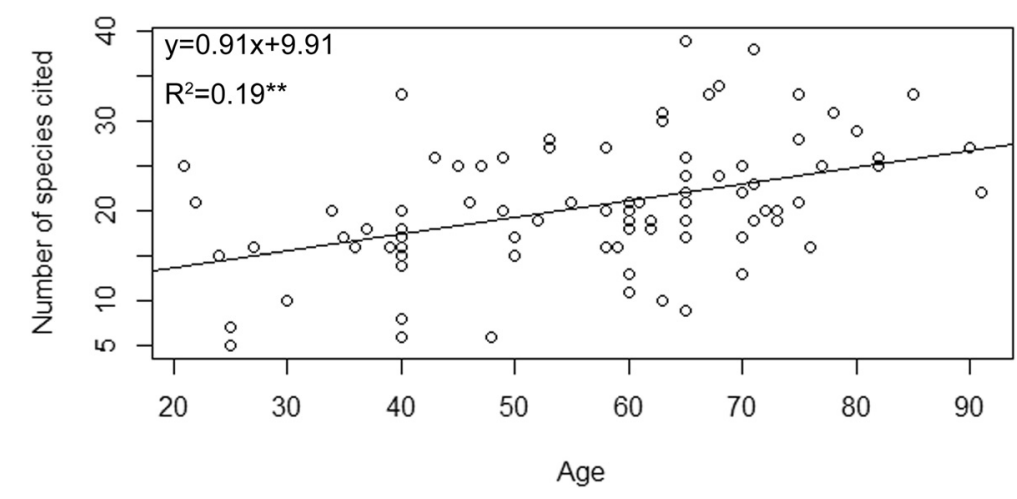

Fig. 6 The relationship between informant age and number of species cited. $\left(R^{2}=\right.$ coefficient, and ${ }^{* *}$ means $P$ value is less than 0.01 .) 
[67]. Contrast to that in Baidi there was no clear-cut division of responsibilities for women and men, and they worked together in agriculture, leading to the matched food knowledge between men and women.

\section{Knowledge transmission between Baidi Naxi and outsiders}

According to our informants, two wild edibles, Nasturtium officinale and Prinsepia utilis, were currently consumed, and they represented different ways of knowledge acquisition. For Nasturtium officinale, local people learned the food use from the tourists from Guangdong province, and they spread the knowledge to near villages. For Prinsepia utilis, over the half of informants knew the fruits can be used to extract oil, but only one informant consumed the oil product. Some informants acquired the knowledge from their neighbors, relatives, and friends in nearby villages. There was one local market in Haba village $(26 \mathrm{Km}$ from Baidi village) for local people around to exchange goods, where also was a site for friends union and information dissemination. Since, the relatives play an important role as transmitters of knowledge and markets are significant sites for food knowledge transmission [68, 69], knowing who holds the traditional knowledge and ensuring the path to transmit it is meaningful ways to protect the knowledge.

The local name of the wild edibles was also helpful in recognizing the knowledge transmission pathways. The wild edibles that have a local Naxi name indicate prolonged consumption history, such as Eutrema yunnanense. The species that do not have local Naxi name may be introduced later, e.g. Nasturtium officinale (Xiyang cai, xiyang means western countries in Mandarin, cai means vegetable) and some fungi (muer, niuganjun etc.). It indicates that Naxi people may learn to use them from the Han Chinese as well as other minorities. Compared with another study in Shangri-La, many species utilized by the Naxi also are used by Tibetans [15], which may be one of the evidence of knowledge transmission.

\section{Conclusion}

Baidi village is an excellent example of a rapidly changing village where local traditions compete with modern ways of life. Although many traditions have been lost in the past years, the Naxi in Baidi still preserves most of food traditions, especially the gathering of the wild species.

We documented 173 wild edible plant species representing 76 families 139 genera from our ethnobotanical survey. Some species were traditionally consumed as an important supplement to the diet, particularly during food shortages e.g. Cardamine macrophylla, C. tangutorum and Eutrema yunnanense, which also were potential wild food products with high nutritional value. The age factor significantly differred the traditional knowledge distribution, but there was no significant difference in knowledge between male and female informants. The traditional food knowledge of the Naxi in Baidi is dynamic, affected by social factors and communicated with the outsiders' food knowledge. Overall, this study provides a deeper understanding of the Naxi traditional knowledge on wild edibles. The study suggests some wild edibles might have an interesting dietary constituent, which necessitates further investigation on the nutrition value as well as market opportunities. With scientific evidence on nutrition value and market opportunity, more people will be attracted toward the wild edibles that will help in addressing food security issues along with conservation of traditional knowledge of the aboriginal population.

\section{Competing interests}

The authors declare that they have no competing interests.

\section{Authors' contributions}

WYH and GYF conceived and designed the research. WYH funded this study. GYF and ZY collected the data, and ZY provided the botanical identification. GYF analyzed the data and prepared the manuscript. HHY undertook a critical review of the manuscript. SR reviewed and rewrote the manuscript. All authors read and approved the final manuscript.

\section{Acknowledgments}

Special gratitude is expressed to the families of Xihong He for their kind hospitality, and to the Jinfu He for his genuine assistance. We are thankful to the families Cuiliu Yang for their helpful comments and suggestions. We are also thankful for the help of other members of our group (Liji He, Zhenzhen Chai, Shan Li, Ruyan Fan). We also thank Renbin Zhu and Dahai Zhu for providing pictures of Cardamine tangutorum and Eutrema yunnanense. This research was funded by National Nature Science Foundation of China (31270379). Sailesh Ranjitkar is supported by the CGIAR research programs on 'Forests, Trees and Agroforestry' (CRP 6.2).

\section{Author details}

${ }^{1}$ Key Laboratory of Economic Plants and Biotechnology, Kunming Institute of Botany, Chinese Academy of Sciences, Kunming 650201, China. ${ }^{2}$ University of Chinese Academy of Sciences, Beijing 100049, China. ${ }^{3}$ World Agroforestry Centre East and Central Asia, Kunming 650201, China. ${ }^{4}$ College of Bioscience and Biotechnology, Yangzhou University, Yangzhou 225009, China.

Received: 14 July 2015 Accepted: 26 January 2016

Published online: 05 February 2016

\section{References}

1. Łuczaj Ł, Köhler P, Pirożnikow E, Graniszewska M, Pieroni A, Gervasi T. Wild edible plants of Belarus: from Rostafiński's questionnaire of 1883 to the present. J Ethnobiol Ethnomed. 2013;9:21.

2. Lulekal E, Asfaw Z, Kelbessa E, Van Damme P. Wild edible plants in Ethiopia: a review on their potential to combat food insecurity. Afr Focus. 2011;24: $71-121$

3. Cunningham AB. Applied ethnobotany. People, wild plant use and conservation. 1 publth edition. London: Earthscan; 2001.

4. Pardo-de-Santayana M, Tardío J, Blanco E, Carvalho AM, Lastra JJ, San Miguel E, et al. Traditional knowledge of wild edible plants used in the northwest of the Iberian Peninsula (Spain and Portugal): a comparative study. J Ethnobiol Ethnomed. 2007;3:27.

5. Luczaj L, Szymański WM. Wild vascular plants gathered for consumption in the Polish countryside: a review. J Ethnobiol Ethnomed. 2007;3:17.

6. Schunko C, Vogl CR. Organic farmers use of wild food plants and fungi in a hilly area in Styria (Austria). J Ethnobiol Ethnomed. 2010;6:17.

7. Rajbhandary S, Ranjitkar S. Herbal drugs and pharmacognosy - monographs on commercially important medicinal plants of Nepal. Kathmandu: Ethnobotanical society of Nepal; 2006. 
8. Pieroni A. Medicinal plants and food medicines in the folk traditions of the upper Lucca Province, Italy. J Ethnopharmacol. 2000;70:235-73.

9. Signorini MA, Piredda M, Bruschi P. Plants and traditional knowledge: an ethnobotanical investigation on Monte Ortobene (Nuoro, Sardinia). J Ethnobiol Ethnomed. 2009;5:6.

10. Grasser S, Schunko C, Vogl CR. Gathering "tea" - from necessity to connectedness with nature. Local knowledge about wild plant gathering in the Biosphere Reserve Grosses Walsertal (Austria). J Ethnobiol Ethnomed. 2012:8:31.

11. González JA, García-Barriuso M, Amich F. The consumption of wild and semi-domesticated edible plants in the Arribes del Duero (SalamancaZamora, Spain): an analysis of traditional knowledge. Genet Resour Crop Evol. 2011:58:991-1006.

12. Kang YX, Luczaj L, Kang J, Wang F, Hou JJ, Guo QP. Wild food plants used by the Tibetans of Gongba Valley (Zhouqu county, Gansu, China). J Ethnobiol Ethnomed. 2014;10(1):20.

13. Xin T, De Riek J, Guo H, Jarvis D, Ma LJ, Long CL. Impact of traditional culture on Camellia reticulata in Yunnan, China. J Ethnobiol Ethnomed. 2015;11(1):1-11.

14. Zhang LL, Zhang Y, Pei SJ, Geng YF, Wang C, Yuhua W. Ethnobotanical survey of medicinal dietary plants used by the Naxi People in Lijiang Area, Northwest Yunnan, China. J Ethnobiol Ethnomed. 2015;11(1):40.

15. Ju Y, Zhuo JX, Liu B, Long CL. Eating from the wild: diversity of wild edible plants used by Tibetans in Shangri-la region, Yunnan, China. J Ethnobiol Ethnomed. 2013;9:28.

16. Yang LX, Ahmed S, Stepp J, Mi K, Zhao YQ, Ma JZ, et al. Comparative homegarden medical ethnobotany of Naxi healers and farmers in Northwestern Yunnan, China. J Ethnobiol Ethnomed. 2014;10(1):6.

17. Liu YC, Dao ZL, Yang CY, Liu YT, Long CL. Medicinal plants used by Tibetans in Shangri-la, Yunnan, China. J Ethnobiol Ethnomed. 2009:5:15.

18. Huber FK, Ineichen R, Yang YP, Weckerle CS. Livelihood and conservation aspects of non-wood forest product collection in the Shaxi Valley, Southwest China. Econ Bot. 2010;64:189-204.

19. He JQ, He JR. The cultural transmission and religion harmony for multivariant nationalities — the Eryueba among the Naxi in Baidi village, Shangri-La as a case study. Thinking. 2009:S1:4-8.

20. Yang FQ. A brief history of modern Naxi Yunnan. Kunming: Yunnan People's Publishing House; 2012.

21. Rock JFC. The ancient Nakhi kingdom of southwest China. Cambridge: Harvard University Press; 1947.

22. Menendez-Baceta G, Aceituno-Mata L, Tardío J, Reyes-García V, Pardo-deSantayana M. Wild edible plants traditionally gathered in Gorbeialdea (Biscay, Basque Country). Genet Resour Crop Evol. 2012;59(7):1329-47.

23. Heckathorn DD. Snowball versus respondent-driven sampling. Sociol Methodol. 2011:41:355-66.

24. Blernackl P, Waldorf D. Snowball sampling: problems and techniques of chain referral sampling. Sociol Methods Res. 1981:10:141-63.

25. Flora of China Editorial Committee. Flora of China. Beijing: Science Press, and St. Louis: Missouri Botanical Garden Press; 1994-2013.

26. Ladio AH, Lozada M. Nontimber forest product use in two human populations from northwest Patagonia: a quantitative approach. Hum Ecol. 2001;29:367-80.

27. Tardío J, Pardo-de-Santayana M. Cultural importance indices. a comparative analysis based on the useful wild plants of southern Cantabria (Northern Spain). Econ Bot. 2008:62:24-39.

28. Pieroni A. Evaluation of the cultural significance of wild food botanicals traditionally consumed in Northwestern Tuscany, Italy. J Ethnobiol. 2001;21: 89-104

29. Calabuig EL (Coord.). Arribes del Duero: Guia de la naturaleza. Leon: Edilesa; 2008.

30. Zeghichi S, Kallithraka S, Simopoulos AP, Kypriotakis Z. Nutritional composition of selected wild plants in the diet of Crete. World Rev Nutr Diet. 2003;91:22-40.

31. Wang XM, Li J, Li ZX, Zhao WQ. Antioxidant activity of extracts of Laolongpi in vitro. Mod Tradit Chin Med. 2010;30:79-80.

32. Johns T, Chapman L. Phytochemicals ingested in traditional diets and medicines as modulators of energy metabolism. In: Arnason JT, Mata R, Romeo JT, editors. Phytochemistry of medicinal plants. New York: Plenum Press; 1995. p. 161-88.

33. Boa E. Wild edible fungi: a global overview of their use and importance to people. Rome: FAO; 2004
34. Johns T. With bitter herbs they shall eat it: chemical ecology and the origins of human diet and medicine. Tucson: The University of Arizona Press; 1990.

35. Balick MJ, Cox PA. Plants, people and culture, the science of ethnobotany. New York: Scientific American Library; 1996.

36. Jagtap SS, Satpute RA, Rahatgaonkar AM, Lanjewar KR. Phytochemical screening, antioxidant, antimicrobial and quantitative multi-elemental analysis of Habenaria longicorniculata J. Graham. J Acad Ind Res. 2014;3: 108-17.

37. Rajendran A, Rao NR, Kumar KR, Henry AN. Some medicinal orchids of southern India. Anc Sci Life. 1997;17:10-4.

38. Dogan Y, Ugulu I, Durkan N. Wild edible plants sold in the local markets of Izmir, Turkey. Pak J Bot. 2013:45:177-84.

39. Kumar A. Ethnobotanical study of wild vegetables used by rural communities of Kannauj district, Uttar Pradesh, India. Emir J Food Agric. 2013;25:760-6.

40. Do Nascimento VT, De Lucena RFP, Maciel MIS, De Albuquerque UP. Knowledge and use of wild food plants in areas of dry seasonal forests in Brazil. Ecol Food Nutr. 2013;52:317-43.

41. Blanco E. Etnobotánica o la respuesta a nuestras necesidades. La naturaleza: tradiciones del entorno vegetal; 2000.

42. Lv C, Pu ZH, Yin ZQ, Li C, Du YH. Study on the anti-bacteria effect of extracts from Prinsepia utilis Royle Oil Meal in vitro. J Anhui Agric Sci. 2009; 37:10533-5.

43. Zhan SQ, Yuan DS, Li XT, Li JL, Yin ZQ. Identification and determination of total flavonoid: from Prinsepia utilis Royle. Med Plant. 2010;1:12-5.

44. Chen AJ, Li CH, Gao WH, Hu ZD, Chen XG. Separation and determination of active components in Schisandra chinensis Baill. and its medicinal preparations by non-aqueous capillary electrophoresis. Biomed Chromatogr. 2005; 19:481-7

45. Li RT, Sun HD. Studies on the chemical constituents and bioactivities of five Schisandra medicinal species and Elsholtzia bodinieri. J Grad Sch Chin Acad Sci. 2008:25:569-75.

46. Xu YQ, Yao Z, Hu JY, Teng J, Takaishi Y, Duan HQ. Immunosuppressive terpenes from Prinsepia utilis. J Asian Nat Prod Res. 2007;9:637-42.

47. Bhattarai NK. Medical ethnobotany in the Karnali Zone, Nepal. Econ Bot. 1992;46:257-61.

48. Li SL, Yu DP, Li CH, Xie KP, Qi DJ. Development and utilization of Cardamine macrophylla Wild. Resour Dev Market. 2010;26:139-40.

49. Dong WW, Lu ZC, Qu Y, Lu RH. Study on nutritional components of Cardamine macrophylla wild. Nat Prod Res Dev. 2007;19:442-4.

50. Xiang JQ, Li YJ, Yang YK, Long L, Yin HQ, Shai CQ, et al. Research stutus of Cardimine. J Hubei Univ Nationalities (Natural Science Edition). 2011; 29: $440-3$

51. Kang YX, Łuczaj Ł, Ye S, Zhang SJ, Kang J. Wild food plants and wild edible fungi of Heihe valley (Qinling Mountains, Shaanxi, central China): herbophilia and indifference to fruits and mushrooms. Acta Soc Bot Pol. 2012;81:405-13.

52. Wang LX. Exploitation and utilization on wild vegetable resources in Huangshan region. Resour Dev Market. 2006;22:469-71.

53. Vainio-Mattila K. Wild vegetables used by the Sambaa in the Usambara Mountains, NE Tanzania. Ann Bot Fennici. 2000;37:57-67.

54. Kayang $\mathrm{H}$. Tribal knowledge on wild edible plants of Meghalaya, Northeast India. Indian J Tradit Know. 2007:6:177-81.

55. Łuczaj $Ł$. Changes in the utilization of wild green vegetables in Poland since the 19th century: a comparison of four ethnobotanical surveys. J Ethnopharmacol. 2010;128:395-404.

56. Pemberton RW, Lee NS. Wild food plants in South Korea; market presence, new crops, and exports to the United States. Econ Bot. 1996;50:57-70.

57. Łuczaj Ł. Ethnobotanical review of wild edible plants of Slovakia. Acta Soc Bot Pol. 2012:81:245-55

58. Lu LH, Guo Q, Yan WL, Wu DC, Liu XF, Fan MR, et al. Study on volatile components of Yunnan Eutrema Wasabi Maxim. Yunnan Chem Technol. 2011;38:18-20

59. Zhang JJ, He FF. Study on the callus and cells' suspension culture on Eutrema Wasabia and the flavor components. Pharm Biotechnol. 2011;18:21-5.

60. Etkin NL. Medicinal cuisines: diet and ethnopharmacology. Int Pharmacogn. 1996;34:313-26.

61. Rigat M, Bonet MÀ, Garcia S, Garnatje T, Vallès J. Ethnobotany of food plants in the high river Ter valley (Pyrenees, Catalonia, Iberian Peninsula): non-crop food vascular plants and crop food plants with medicinal properties. Ecol Food Nutr. 2009;48:303-26 
62. Kang YX, Łuczaj $Ł$, Kang J, Zhang SJ. Wild food plants and wild edible fungi in two valleys of the Qinling Mountains (Shaanxi, central China). J Ethnobiol Ethnomed. 2013;9(1):26.

63. Quinlan MB, Quinlan RJ. Modernization and medicinal plant knowledge in a caribbean horticultural village. Med Anthropol Q. 2007;21:169-92.

64. Pfeiffer J, Butz R. Assessing cultural and ecological variation in ethnobiological research: the importance of gender. J Ethnobiol. 2005;25: 240-78.

65. Panyaphu K, Van On T, Sirisa-ard P, Srisanga P, Kaow SC, Nathakamkitkul S. Medicinal plants of the Mien (Yao) in northern Thailand and their potential value in the primary healthcare of postpartum women. J Ethnopharmacol. 2011;135:226-37.

66. Junsongduang A, Balslev H, Inta A, Jampeetong A, Wangpakapattanawong P. Karen and Lawa medicinal plant use: Uniformity or ethnic divergence? J Ethnopharmacol. 2014;151:517-27.

67. Garibay-Orijel R, Ramírez-Terrazo A, Ordaz-Velázquez M. Women care about local knowledge, experiences from ethnomycology. J Ethnobiol Ethnomed. 2012;8:25.

68. Haselmair R, Pirker $H$, Kuhn E, Vogl CR. Personal networks: a tool for gaining insight into the transmission of knowledge about food and medicinal plants among Tyrolean (Austrian) migrants in Australia, Brazil and Peru. J Ethnobiol Ethnomed. 2014:10:1.

69. Powell B, Ouarghidi A, Johns T, Tattou MI, Eyzaguirre P. Wild leafy vegetable use and knowledge across multiple sites in Morocco: a case study for transmission of local knowledge? J Ethnobiol Ethnomed. 2014;10:34.

\section{Submit your next manuscript to BioMed Central and we will help you at every step:}

- We accept pre-submission inquiries

- Our selector tool helps you to find the most relevant journal

- We provide round the clock customer support

- Convenient online submission

- Thorough peer review

- Inclusion in PubMed and all major indexing services

- Maximum visibility for your research

Submit your manuscript at www.biomedcentral.com/submit

C) Biomed Central 